\title{
A Survey Of Earth-Moon Libration Orbits: Stationkeeping Strategies And Intra-Orbit Transfers
}

\author{
David Folta ${ }^{*}$ and Frank Vaughn ${ }^{\#}$ \\ NASA Goddard Space Flight Center \\ Greenbelt, MD 20771
}

\begin{abstract}
Cislunar space is a readily accessible region that may well develop into a prime staging area in the effort to colonize space near Earth or to colonize the Moon. While there have been statements made by various NASA programs regarding placement of resources in orbit about the Earth-Moon Lagrangian locations, there is no survey of the total cost associated with attaining and maintaining these unique orbits in an operational fashion. Transfer trajectories between these orbits required for assembly, servicing, and positioning of these resources have not been extensively investigated. These orbits are dynamically similar to those used for the Sun-Earth missions, but differences in governing gravitational ratios and perturbation sources result in unique characteristics.

We implement numerical computations using high fidelity models and linear and nonlinear targeting techniques to compute the various maneuver $\Delta \mathrm{V}$ and temporal costs associated with orbits about each of the Earth-Moon Lagrangian locations $\left(\mathrm{L}_{1}, \mathrm{~L}_{2}, \mathrm{~L}_{3}, \mathrm{~L}_{4}\right.$, and $L_{5}$ ). From a dynamical system standpoint, we speak to the nature of these orbits and their stability. We address the cost of transfers between each pair of Lagrangian locations.
\end{abstract}

\footnotetext{
* Senior Aerospace Engineer, Flight Dynamics Analysis Branch, NASA Goddard Space Flight Center, Greenbelt, MD, 20771, Code 595

\# Aerospace Engineer, Flight Dynamics Analysis Branch, NASA Goddard Space Flight Center, Greenbelt, MD, 20771, Code 595
} 


\section{Introduction}

With the recent announcement of the Vision for Space Exploration, ready access to Cislunar space has become vital to achieving future space exploration goals. Missions may be established in Earth-Moon libration orbits as a part of communication architecture or to deploy and service spacecraft either in transit from the Moon or stationed at other Sun-Earth or Earth-Moon libration regions. To understand the costs associated with these missions, a survey of stationkeeping about all Earth-Moon libration points and the $\Delta \mathrm{Vs}$ to transfer between them is investigated. Analysis suggests these costs can range from centimeters per second per year for the more stable orbits of the co-linear $\mathrm{L}_{3}$, and triangular $\mathrm{L}_{4}$ and $\mathrm{L}_{5}$ locations to nearly tens, possibly hundreds of meters per second for the unstable co-linear $\mathrm{L}_{1}$ and $\mathrm{L}_{2}$ locations. Of course stationkeeping cost depends on several parameters, such as orbit amplitudes (as measured in a rotating, libration centered coordinate system), mismodeled accelerations due to solar radiation pressure and third body gravity, the calibration of the propulsion system, and the accuracy of the post maneuver navigation solutions. Our analysis incorporates these errors into both linear and non-linear control efforts to estimate the yearly stationkeeping cost for all the Earth-Moon libration orbits. We also address the $\Delta \mathrm{V}$ cost of transfers between these orbit locations.

\section{A. Initial Conditions}

Figure 1 presents the orbit geometries of interest and orients the reader to the coordinate system used in the simulations. All orbital parameters are stated in the Earth-Moon Rotating Libration Point (RLP) coordinate system, where the line from the Earth to the Moon represents the $+x$-axis, the velocity direction of the Moon the $+y$-axis and the $+z$-axis completes the triad. Both Lissajous and halo (periodic) orbits are surveyed. The orbital parameters for the halo orbit are computed from the dynamics of the region and the equations of motion where the out-of-plane and in-plane frequencies are equal. ${ }^{1}$ For our initial conditions, we solved the frequency equations for the $\mathrm{z}$-axis component versus the $\mathrm{x}$-axis component. This solution allows us to initialize the orbits along the $\mathrm{x}$-axis so that we can input the initial state simply as the $\mathrm{x}$ and $\mathrm{z}$-axes positions and the $\mathrm{y}$-axis velocity with all other components set to zero. Figure 2 shows this relationship between in-plane $\mathrm{x}$-amplitude and out-of-plane z-amplitude for the Earth-Moon system by solving

$$
\begin{aligned}
& \omega_{x}=0.1387811 *(\text { Emass } / \text { Mmass })^{2}+0.04349909 * A y^{2}-0.04060812 * A z^{2} \\
& \omega_{x}=0.5981779 *(\text { Emass } / \text { Mmass })^{2}-0.03293845 * A y^{2}+0.03923249 * A z^{2} \\
& \text { with } f=1.865485 *\left(\left(1+M^{2}\right) * \omega_{z}{ }^{2}\right)-1.794291 *\left(\left(1+M^{2}\right) * \omega_{x}{ }^{2}\right)
\end{aligned}
$$

where Emass/Mmass is the Earth to Moon mass ratio and $\mathrm{M}$ is the non-dimensionalized lunar mass. Table 1 provides the initial RLP centered coordinates of the co-linear and triangular libration orbits surveyed.

Table 1. Earth Moon Libration Orbit Initial Conditions

\begin{tabular}{|c|c|c|c|c|}
\hline $\begin{array}{c}\text { Libration } \\
\text { Orbit } \\
\text { Location }\end{array}$ & $\begin{array}{c}\text { Small } \\
\text { Lissajous } \\
x, y, z(\mathrm{~km})\end{array}$ & $\begin{array}{c}\text { Large Lissajous } \\
x, y, z(\mathrm{~km})\end{array}$ & $\begin{array}{c}\text { Small Halo } \\
x, y, z(\mathrm{~km})\end{array}$ & $\begin{array}{c}\text { Large Halo } \\
x, y, z(\mathrm{~km})\end{array}$ \\
\hline $\mathrm{L}_{1}, \mathrm{~L}_{2}, \mathrm{~L}_{3}$ & 5000 & 10,000 & 5120 & 10,000 \\
& 15000 & 25,000 & 15000 & 25,000 \\
& 2000 & 15,000 & 5000 & 10,000 \\
\hline $\mathrm{L}_{4}$ and $\mathrm{L}_{5}$ & 2000 & 45,000 & $\mathrm{n} / \mathrm{a}$ & $\mathrm{n} / \mathrm{a}$ \\
(no z component) & 2000 & 45,000 & & \\
\hline
\end{tabular}




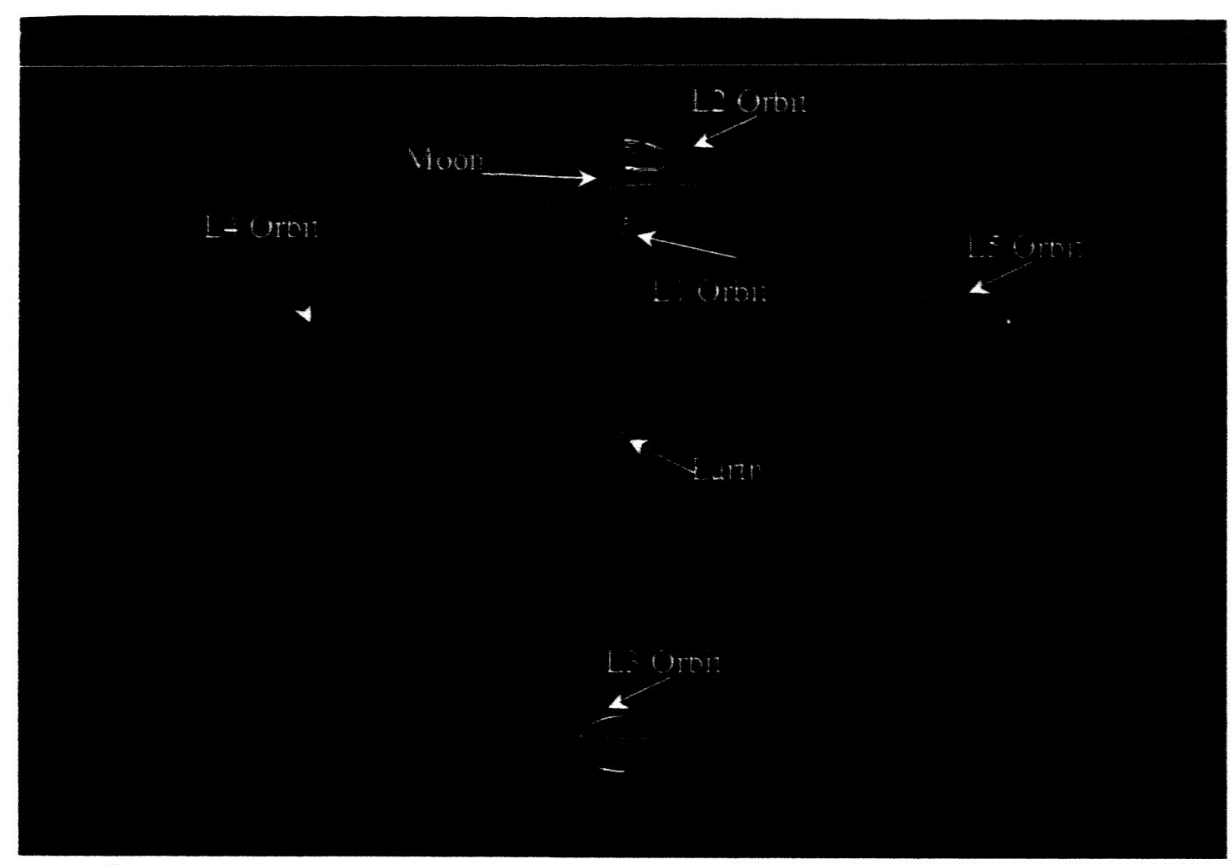

Figure 1. A View Of All Libration Orbits In The Earth-Moon System

\section{B. Historical Overview and Previous Work}

There are numerous references for the discussion of stability and control for both co-linear and triangular locations. In the 1960 s, Steg and Michael provided analysis of the stability of triangular regions and a discussion of the motion of a particle placed at the Earth-Moon $\mathrm{L}_{4}$ location. ${ }^{2,3}$ They investigated the influence of third body perturbations and an elliptical lunar orbit was introduced. Farquhar and Hoffman provided analysis and discussion of stability and control of the Earth-Moon co-linear $\mathrm{L}_{2}$ and $\mathrm{L}_{1}$ locations, respectively, in a classical control theory or linear approximation while more recently Scheeres provides a statistical analysis approach. ${ }^{4,5}$ Howell and Keeter addressed the use of selected maneuvers to

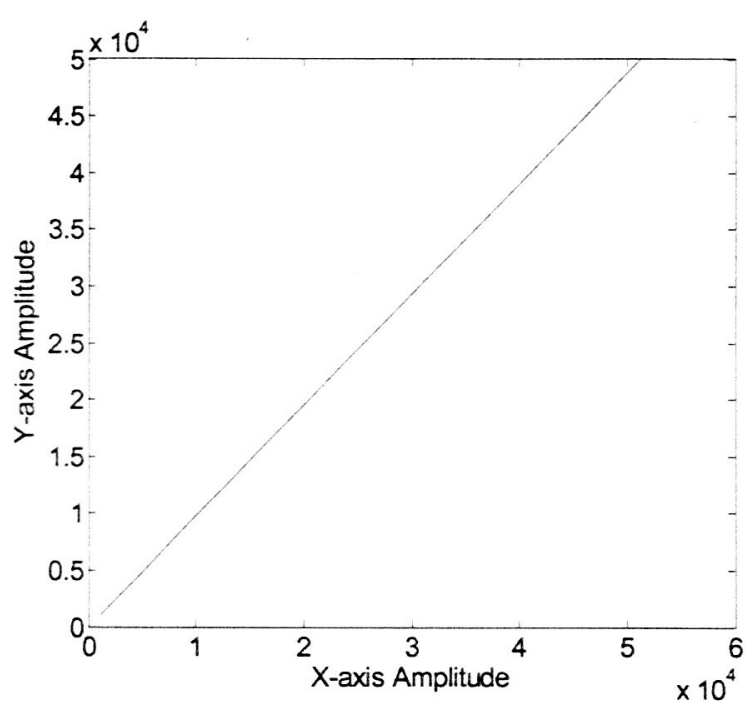

Figure 2. Earth-Moon X-Amplitude vs. ZAmplitude for Equal Frequencies

eliminate the unstable modes or a reference orbit. ${ }^{7}$ Of course, centuries ago, others provided the basics of the dynamics of these locations, such as Euler, Lagrange, and Jacobi. The Jacobi integral will be used herein for a discussion of the stability of triangular locations and transfer trajectories from triangular locations to co-linear locations. ${ }^{8}$ Howell provides an excellent discussion of stability including the Eigenstructures in the Sun-Earth locations. ${ }^{9}$ Folta, et al provides an analysis of transfers between the Sun-Earth locations and the Earth-Moon locations. ${ }^{10}$ 


\section{Dynamics Of The Earth-Moon Region}

The equations of motion of a spacecraft in the Earth-Moon region are similar in nature to those of the Sun-Earth system. ${ }^{3,4,5}$ The Earth-Moon mass ratio of approximately 0.012 is the major difference. From this information, one can construct the Circular Restricted Three Body (CRTB) process matrix or state Jacobian at the libration point using the pseudopotential. Unfortunately doing so does not allow the analyst to include the effects of lunar eccentricity, solar gravity, and solar radiation pressure directly into the state integration process. Past efforts included the use of disturbance accommodation techniques to eliminate a control effort to address natural motion. ${ }^{5}$ The generation of our initial state and the integration of our states use full ephemeris models and not analytical approximations. As numerous references are available on the derivation of the equations of motion they will not be discussed here. To use full ephemeris modeling in the controller design, we use the process matrix estimates at the end of each integration output interval. ${ }^{11}$ This interval is a user defined interval and was selected as $1 / 4$ orbit for our cases.

The eigenstructure of the libration orbits about $\mathrm{L}_{1}$ and $\mathrm{L}_{2}$ can be constructed to give the reader a sense of the stable and unstable directions in the Earth-Moon system, obviously important in stationkeeping. Figure 3 presents the stable and unstable modes associated with the Earth-Moon system for a specified $\mathrm{L}_{1}$ orbit. The motion of a spacecraft is dependent upon the dynamical nature of the region for its orbit stability and this influences the methods for transfer between the libration points. The goal in our analysis is to minimize the cost, while using realistic time transfers of less than 28 days. In addition to the eigenstructure, the Jacobi constant can also be used to visualize the possible paths of transfers and motion about the more stable locations Earth-Moon $\mathrm{L}_{3}, \mathrm{~L}_{4}$, and $\mathrm{L}_{5}{ }^{1}$. Figure 4 presents the lines of zero velocity for the Earth-Moon region. These curves bound the motion for a given Jacobi constant (C) with values of 2.991 to 3.2 shown, with the lower $\mathrm{C}$ values at $\mathrm{L}_{4}$ and $\mathrm{L}_{5}$.

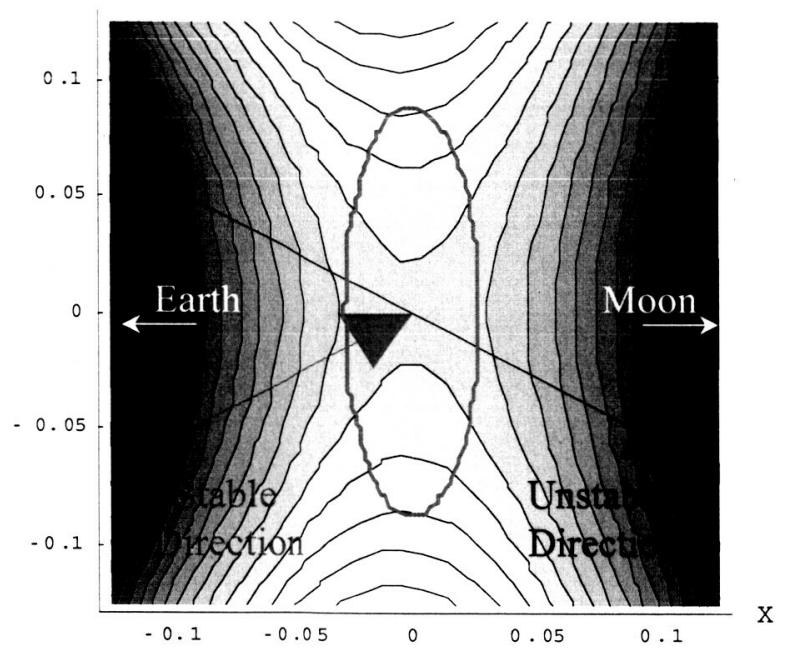

Figure 3. Stable and Unstable Earth-Moon Directions $\left(\mathrm{L}_{1}\right)$

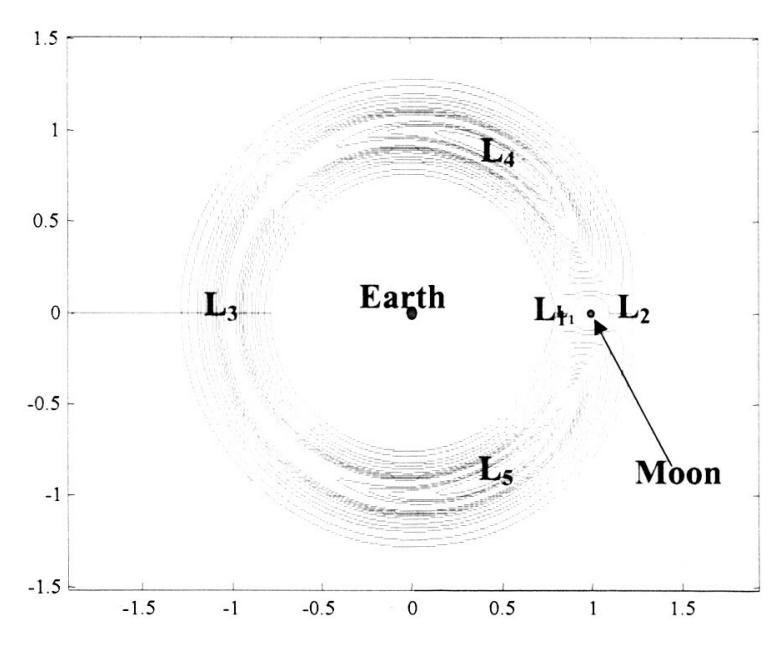

Figure 4. Lines of Zero Velocity

\section{A. Modeling and conditions}

A full ephemeris model is used with point mass Earth, Moon, and Sun gravity, solar radiation pressure based on a spacecraft area of $5 \mathrm{~m}^{2}$, a mass of $1000 \mathrm{~kg}$, and use of DE405 files for the location of each body. Our integration uses a variable step Runge-Kutta 8/9 with pulsating coordinates. The pulsating coordinate system is formulated from the time dependent position of 
the Earth and Moon calculated at each integration step. The libration point locations are also calculated at the same integration interval.

\section{B. Numerical Models: Discrete Linear Quadratic Regulator and Differential Correction}

In addition to the numerically calculated $\triangle \mathrm{Vs}$, MATLAB Simulink is used for an implementation of discrete Linear Quadratic Regulator (dLQR) control. The dLQR was implemented to compare and contrast the stability and control required using discrete thrust applied about the orbit for stationkeeping to that of an operational environment where maneuvers are limited in location and time. A discrete controller is useful in assessing the stationkeeping $\Delta \mathrm{V}$ since it provides consistency with previous work that used linear analysis. ${ }^{3,4,5}$ A continuous controller could have been used as well, but continuous methods are not used in the operational environment since maneuver planning is not just a function of trajectory correction needs, but also on spacecraft operations, science operations, momentum management, and communication requirements to name a few. The MATLAB simulation is shown in Figure 5.

\section{B.1. MATLAB Simulink - Discrete LQR}

The dLQR method implemented is the standard method that is based on linearization of the system and the use of an algebraic Riccati equation to solve for the gain. ${ }^{12}$ A continuous control can be computed using linear dynamics derived from the mean motion and mass ratios of the two primary and secondary bodies, but it does not include the perturbations and ephemeris in the dynamics. For our discrete LQR control, several modifications were made. To compute a linear dynamics model, a multiple revolution numerically stable orbit was computed in GSFC's Swingby program. The initial velocity conditions were changed to balance the orbit through a differential correction process to the $\Delta \mathrm{V}$ accuracy of the processor, approximately $1.0 \mathrm{e}-16$. The modeling includes the point mass perturbations of the Earth, Moon, Sun, solar radiation pressure, and major planets, sometimes referred to as using full ephemeris, and includes the lunar and planetary eccentricity. From this numerically balanced orbit, several matrices may be calculated: the process matrix or state Jacobian, the state transition matrix (STM), and the monodromy matrix. For our simulation, the process matrix was used to represent the tracking error dynamics. The process matrix and state transition matrix where calculated on the reference orbit by the Generator program developed by Purdue University. These matrices were then used at a userdefined interval (we updated these matrices at intervals between 1 and 3.5 days) as input to the $\mathrm{dLQR}$ process. After each update, the Riccati equation was solved to obtain the discrete control gain assuming a zero-order hold. To simulate the controller we used a slight mismodeling of $3 \%$ in the SRP for the controlled orbit and trajectory errors. This mismodeling of the solar radiation pressure is consistent with results observed by the GSFC Flight Dynamics Facility. The navigation errors were assumed to have a uniform random distribution with a limit less than 1 $\mathrm{cm} / \mathrm{s}$ in each direction. Navigation errors were applied after each maneuver. The errors in maneuver magnitude were modeled at $2 \%$ in both positive and negative maneuver directions. We used diagonal control weights of $1 \mathrm{e}-11$, position weights of $1 \mathrm{e}-2$, and velocity weights of $1 \mathrm{e}-4$ for the dLQR. 


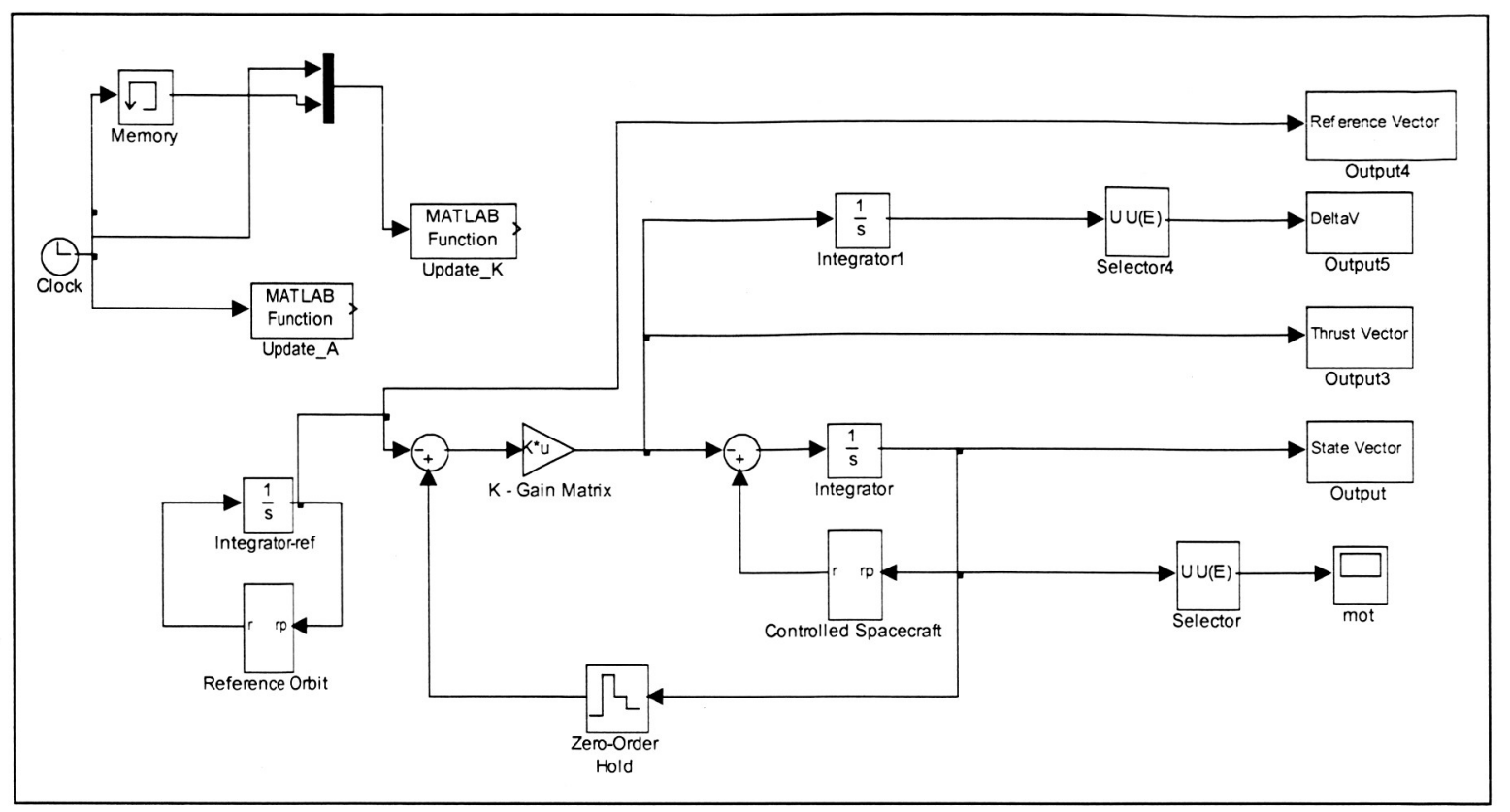

Figure 5. MATLAB Simulink Setup for dLQR Stationkeeping

\section{B.2. Numerical Targeting - Differential Correction}

The Differential Correction (DC) scheme is based on the construction of an invertible sensitivity matrix by numerical sampling achieved goals for given initial velocity perturbations. ${ }^{13} \mathrm{~A}$ simple matrix multiplication is performed to compute the new variable updates (the stationkeeping maneuver) to achieve the target goals. The DC uses several variables and target goals specific for each orbit class controlled. For example, for a Lissajous orbit all targeting was performed with the goals assigned to the $\mathrm{x}-\mathrm{z}$ plane crossing in the Earth-Moon RLP coordinate system (see Figure 1). ${ }^{14,15}$ For Lissajous orbits, a $\Delta \mathrm{V}$ on the $\mathrm{x}$-axis was targeted that would balance the orbit to achieve another revolution with an associated zero velocity in the $\mathrm{x}$-axis direction. If the orbit was not a single periodic orbit, we deferred to a doubly periodic target (twice around). For the halo orbit classes, an additional impulsive maneuver was performed to retain the $\mathrm{z}$ amplitude of the initial conditions. The location of this additional $\Delta \mathrm{V}$ was adjusted to provide a minimal $\Delta \mathrm{V}$ cost, but a full trade to identify minimum or optimum locations on an orbit-by-orbit basis was not performed.

The differential correction scenario was developed to compare the more frequently published $\mathrm{LQR}$ or $\mathrm{dLQR}$ method to a more realistic operational scenario. A differential correction scheme using Analytical Graphics' Satellite Tool Kit (STK)® Astrogator module and Swingby was implemented with the idea of adding maneuver and navigation errors similar to those for the Simulink scenario, but in an operational mode. The differential correction stationkeeping analysis was performed using Astrogator. The analysis was partially automated using MATLAB ${ }^{\circledR}$ and STK's Connect and MATLAB Interface modules to build stationkeeping scenarios up to 1 year in duration. Stationkeeping of Lissajous orbits was performed impulsively in either of two directions, along the x-axis or the $y$-axis of the Earth-Moon RLP coordinate system. Maneuvers were only allowed to occur at the $\mathrm{x}-\mathrm{z}$ plane crossings and the target at each crossing is a velocity of zero in the $\mathrm{x}$-axis direction of the Earth-Moon RLP coordinate system. This method does not follow a reference orbit but simply tries to keep the spacecraft in an orbit about the libration point while trying to meet the required amplitudes. For the halo orbits the 
strategy is slightly more complicated. It begins with a maneuver in the RLP $x$-axis direction at the initial $x-z$ plane crossing to initially balance the orbit at the next $x-z$ plane crossing. Starting back at this initial location, another maneuver in the $x$-axis direction is applied, the orbit is propagated for approximately 1.5 days, and an additional maneuver is applied in the RLP $\mathrm{z}$-axis direction to achieve an RLP x-axis velocity of zero and a $z$-amplitude consistent with the initial state at the next $x-z$ crossing. At this point a maneuver is applied in the RLP $x$-axis direction to achieve an RLP $x$-axis velocity of zero at the subsequent $x-z$ plane crossing where the entire sequence begins again.

\section{Stationkeeping Results}

Over a restricted range of orbit dimensions (see Table 1), we provide a parametric survey of the total stationkeeping $\Delta \mathrm{V}$ associated with each libration point orbit. A combination of Lissajous and halo orbits for the co-linear locations and a $y$-amplitude for the triangular cases was used to estimate the control efforts. Our analysis uses an impulsive $\Delta \mathrm{V}$ maneuver model. This method is chosen to develop feasible strategies that meet operational limitations. Our initial analysis indicates that one can target to a dozen libration orbit revolutions, but the control precision of the initial velocity to achieve the orbit goals approaches values unattainable by any operational propulsion system. There is also the problem that the dynamics of the problem for multi-rev orbit targeting are sensitive to perturbations on the order of $1 \mathrm{e}-12 \mathrm{~km} / \mathrm{sec}$.

\section{A. Stationkeeping at Unstable Co-linear locations, $L_{1}$ and $L_{2}$}

Tables 2-6 provide the yearly stationkeeping $\Delta \mathrm{V}$ cost along the $\mathrm{x}$-axis and $\mathrm{y}$-axis associated with the initial conditions listed in Table 1 for the DC and dLQR methods. Figures 6 though 13 present selected individual and cumulative $\Delta \mathrm{V}$ stationkeeping costs for the values in bold type in Table 3 . As seen in the figures, the control effort is dependent upon the perturbations included. RLP yaxis control proved to be approximately twice as expensive as $\mathrm{x}$-axis control, which is typical of past experience. Table 6 provides $\Delta \mathrm{V}$ cost for dLQR control.

Table 2. Differential Correction Stationkeeping Yearly Cost with No Errors (m/s)

\begin{tabular}{|l|c|c|c|c|}
\hline & Small Lissajous & Small Halo $^{\#}$ & Large Lissajous & Large Halo $^{\#}$ \\
\hline $\mathrm{L}_{1} \mathrm{x}$ axis control & 15.63 & 132.57 & 61.84 & 211.98 \\
\hline $\mathrm{L}_{1}$ y axis control & 34.47 & 171.99 & 116.09 & 308.74 \\
\hline $\mathrm{L}_{2} \mathrm{x}$ axis control & 13.81 & 184.37 & $73.08(110$ days $)$ & 316.99 \\
\hline $\mathrm{L}_{2}$ y axis control & 22.84 & 197.38 & 227.57 & 380.84 \\
\hline
\end{tabular}

Table 3. Differential Correction Stationkeeping Yearly Cost with Navigation and SRP Errors (No $\Delta V$ errors)(m/s)

\begin{tabular}{|l|c|c|c|c|}
\hline & Small Lissajous & Small Halo $^{\#}$ & Large Lissajous & Large Halo $^{\#}$ \\
\hline $\mathrm{L}_{1} \mathrm{x}$ axis control & $\mathbf{2 2 . 9 0}$ & $\mathbf{1 3 5 . 1 9}$ & $\mathbf{6 7 . 3 7}$ & $\mathbf{2 1 1 . 2 1}$ \\
\hline $\mathrm{L}_{1}$ y axis control & 53.44 & 184.53 & 117.82 & 306.23 \\
\hline $\mathrm{L}_{2} \mathrm{x}$ axis control & $\mathbf{1 7 . 8 6}$ & $\mathbf{1 8 0 . 2 7}$ & $\mathbf{7 2 . 5 4 ( 1 1 0 d )}$ & $\mathbf{3 1 6 . 3 0}$ \\
\hline $\mathrm{L}_{2}$ y axis control & 29.64 & 198.63 & 235.99 & 383.91 \\
\hline$\#-\mathrm{z}$-axis control included (Bold x-axis control data is plotted on next two pages)
\end{tabular}


Table 4. Differential Correction Stationkeeping Yearly Cost with Navigation, SRP, and $+\Delta \mathbf{V}$ Errors $(\mathbf{m} / \mathbf{s})$

\begin{tabular}{|l|c|c|c|c|}
\hline & Small Lissajous & Small Halo $^{\#}$ & Large Lissajous & Large Halo $^{\#}$ \\
\hline $\mathrm{L}_{1} \mathrm{x}$ axis control & 159.48 & 119.45 & $54.90(167 \mathrm{~d})$ & $47.37(60 \mathrm{~d})$ \\
\hline $\mathrm{L}_{1} \mathrm{y}$ axis control & 254.36 & 173.47 & $178.58(273 \mathrm{~d})$ & $63.04(60 \mathrm{~d})$ \\
\hline $\mathrm{L}_{2} \mathrm{x}$ axis control & 26.13 & 305.07 & $198.06(213 \mathrm{~d})$ & 316.21 \\
\hline $\mathrm{L}_{2} \mathrm{y}$ axis control & 41.90 & 279.80 & $58.50(111 \mathrm{~d})$ & 397.83 \\
\hline \#- -z-axis control included
\end{tabular}

Table 5. Differential Correction Stationkeeping Yearly Cost with Navigation, SRP, and $-\Delta$ V Errors $(\mathrm{m} / \mathrm{s})$

\begin{tabular}{|l|c|c|c|c|}
\hline & Small Lissajous & Small Halo $^{\#}$ & Large Lissajous & Large Halo $^{\#}$ \\
\hline $\mathrm{L}_{1} \mathrm{x}$ axis control & 68.06 & 433.57 & $62.83(93 \mathrm{~d})$ & 192.63 \\
\hline $\mathrm{L}_{1}$ y axis control & 95.52 & $88.25(108 \mathrm{~d})$ & $132.81(118 \mathrm{~d})$ & $228.55(146 \mathrm{~d})$ \\
\hline $\mathrm{L}_{2} \mathrm{x}$ axis control & 22.11 & $44.15(66 \mathrm{~d})$ & 165.12 & $46.72(14 \mathrm{~d})$ \\
\hline $\mathrm{L}_{2}$ y axis control & 36.37 & $213.19(272 \mathrm{~d})$ & 179.23 & $47.18(14 \mathrm{~d})$ \\
\hline \#- $\mathbf{z}$-axis control included
\end{tabular}

Table 6. dLQR Stationkeeping Yearly Cost $(\mathrm{m} / \mathrm{s})$

\begin{tabular}{|l|c|c|c|c|}
\hline & Small Lissajous & Small Halo & Large Lissajous & Large Halo \\
\hline $\mathrm{L}_{1}$ no errors & 6.41 & 6.11 & 5.61 & 5.99 \\
\hline $\mathrm{L}_{1}$ with errors & 61.26 & 61.13 & 60.22 & 60.48 \\
\hline $\mathrm{L}_{2}$ no errors & 5.37 & 5.38 & 5.38 & 5.61 \\
\hline $\mathrm{L}_{2}$ with errors & 60.87 & 61.00 & 59.88 & 59.86 \\
\hline
\end{tabular}

The cost of stationkeeping using the dLQR controllers is similar to those reported by Hoffman and Farquhar where for a Lissajous with amplitudes of approximately $10,000 \mathrm{~km}$ in $\mathrm{X}$-axis and $20,000 \mathrm{~km}$ in $\mathrm{z}$-axis the $\Delta \mathrm{V}$ cost per year was $51.4 \mathrm{~m} / \mathrm{s}$ and $23.5 \mathrm{~m} / \mathrm{s}$, respectively. ${ }^{4,5}$ Similarly, for a halo orbit with amplitudes of $1,000 \mathrm{~km}$ in $\mathrm{x}$-axis and $3000 \mathrm{~km}$ in $\mathrm{z}$-axis, they report 132.0 $\mathrm{m} / \mathrm{s}$ and $140.0 \mathrm{~m} / \mathrm{s}$, respectively. The major difference in this work is the inclusion of perturbations and the use of a full ephemeris model. The other references used CRTB with perturbation added separately. In Hoffman, a disturbance accommodation method was used to remove frequencies associated with lunar eccentricity, etc., that the control effort should avoid in minimizing the stationkeeping cost. Note that the DC yields $\Delta \mathrm{V}$ cost lower than the dLQR for Lissajous orbits, as a reference orbit is not followed. The halo orbit DC $\Delta \mathrm{V}$ cost is quite large in comparison. This can be explained by the choice to force the z-amplitude to remain near the initial condition by non-optimal maneuver locations even though the environmental perturbations immediately affect the $\mathrm{z}$-axis value. 


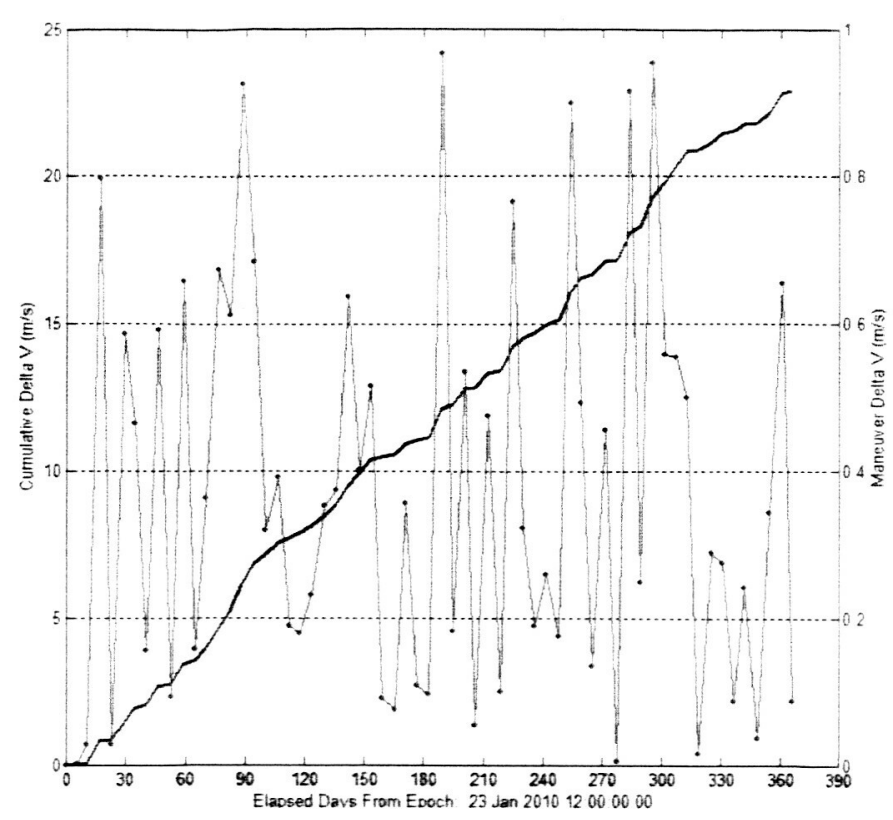

Figure 6: $X$-axis Control, Small $L_{1}$ Lissajous

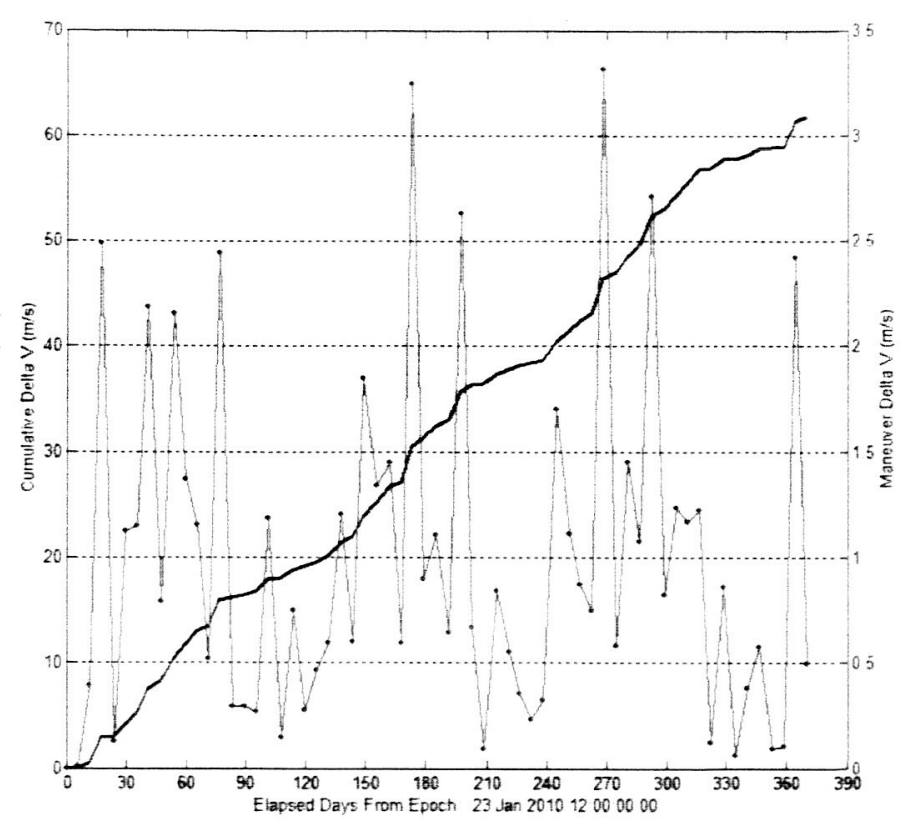

Figure 7: $\mathbf{X}$-axis Control, Large $L_{1}$ Lissajous

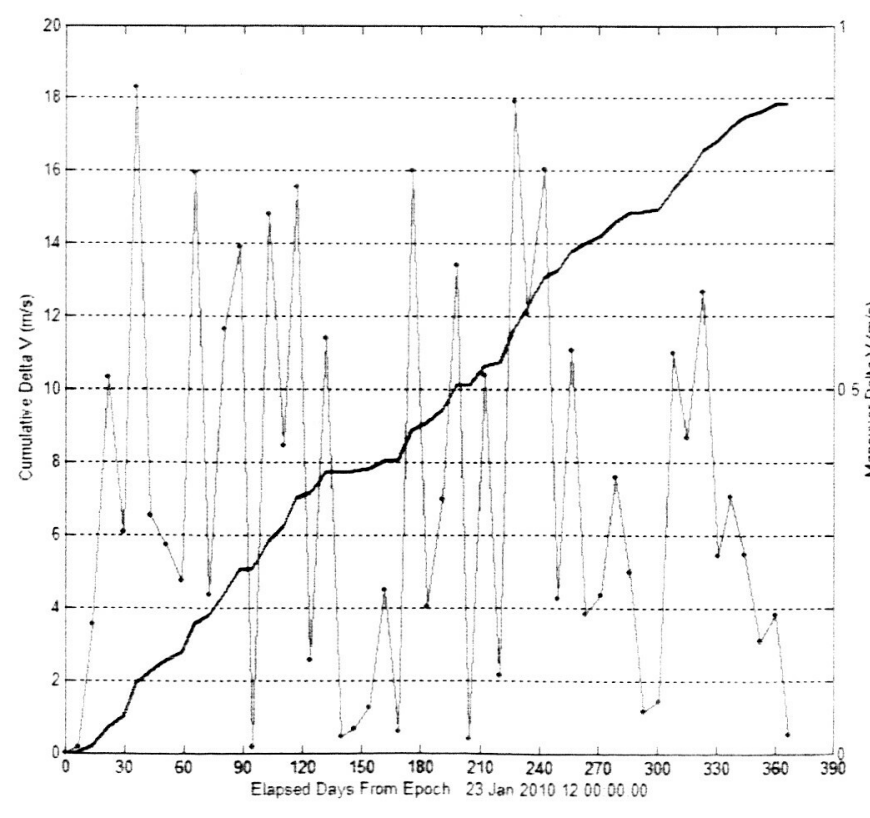

Figure 8: $\mathrm{X}$-axis Control, Small $\mathrm{L}_{2}$ Lissajous

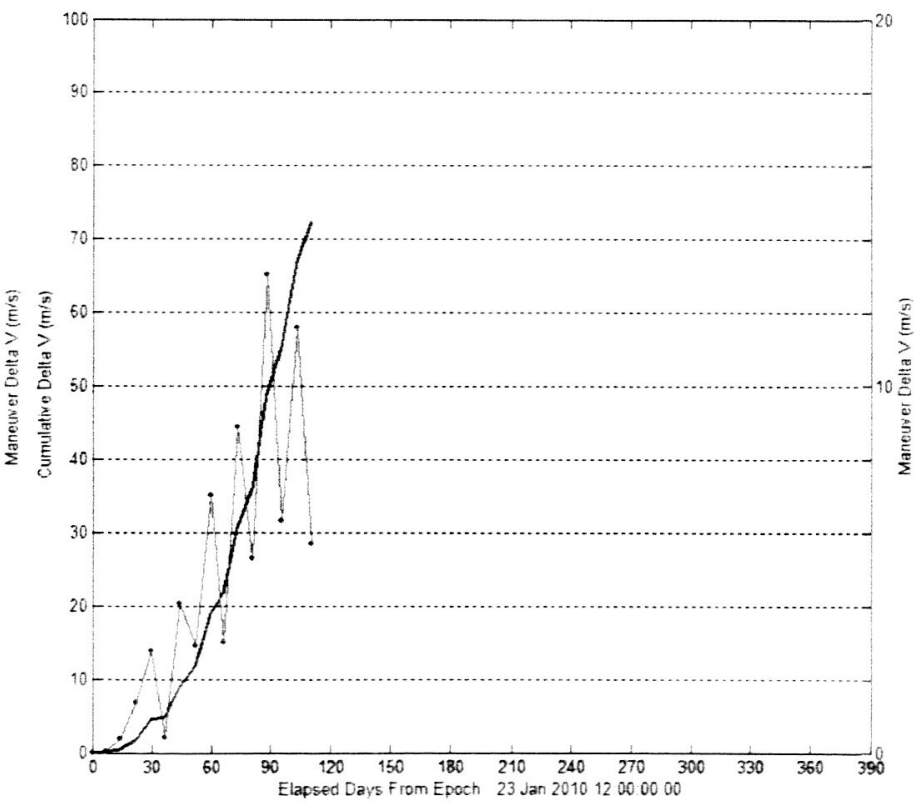

Figure 9: $\mathrm{X}$-axis Control, Large $\mathrm{L}_{2}$ Lissajous 


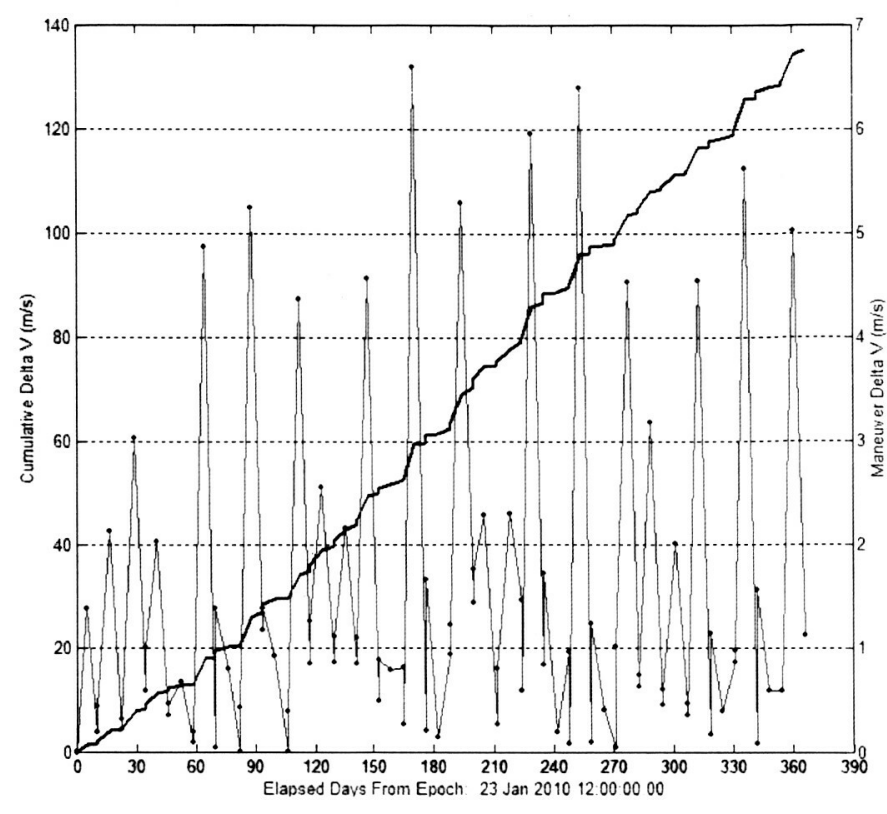

Figure 10: $\mathrm{X}$-axis Control, Small $\mathrm{L}_{1}$ Halo

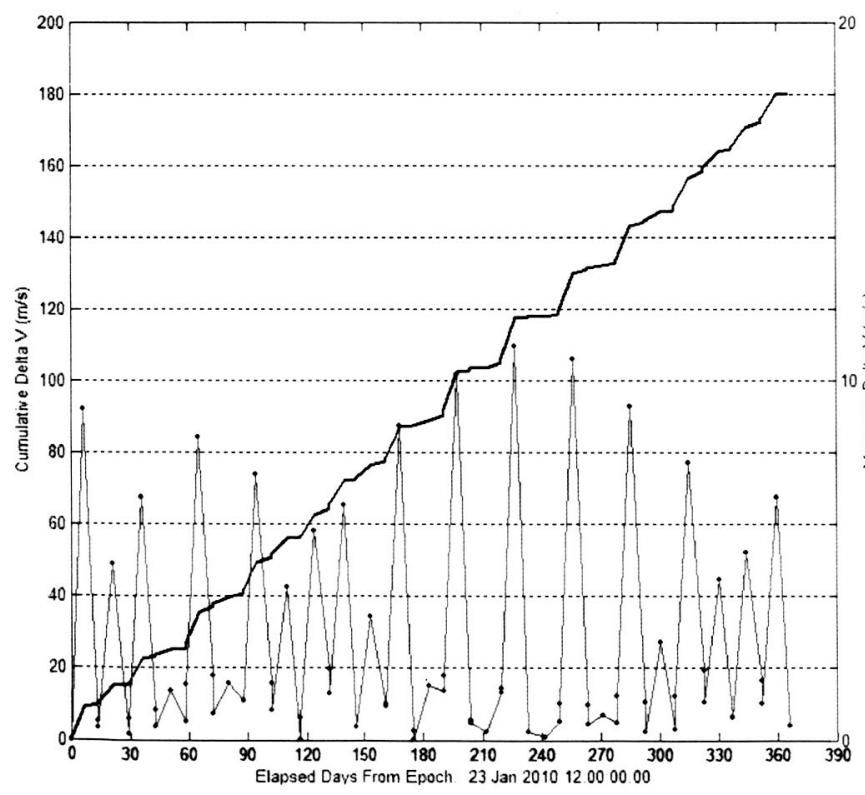

Figure 12: $\mathrm{X}$-axis Control, Small $\mathrm{L}_{2}$ Halo

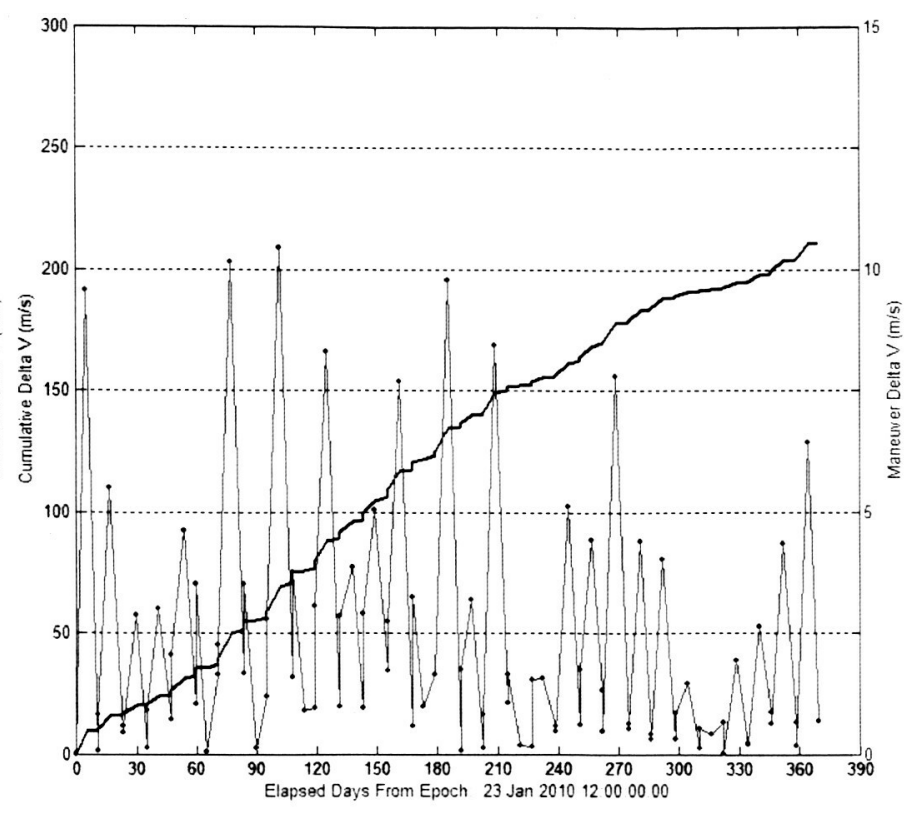

Figure 11: $\mathrm{X}$-axis Control, Large $\mathrm{L}_{1}$ Halo

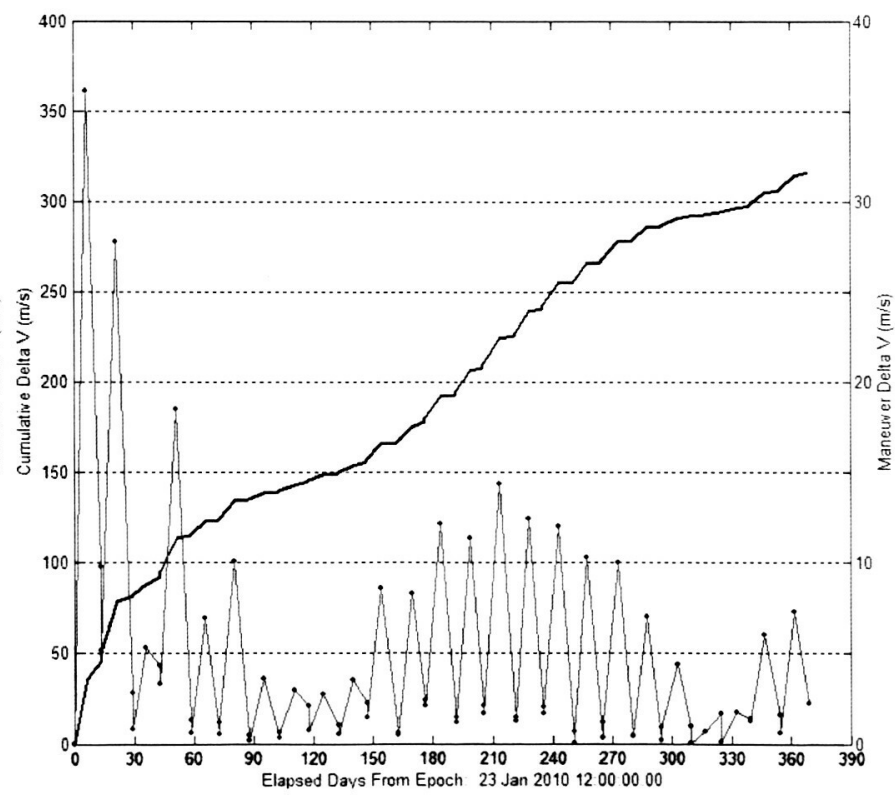

Figure 13: $X$-axis Control, Large $L_{2}$ Halo 


\section{B. Stationkeeping at the $\mathrm{C} 0$-linear $\mathbf{L}_{3}$ point}

Stationkeeping about the Earth-Moon $\mathrm{L}_{3}$ point is unlike stationkeeping about the $\mathrm{L}_{1}$ or $\mathrm{L}_{2}$ locations. This location is quasi-stable in that selection of correct initial velocities can keep the spacecraft in the $\mathrm{L}_{3}$ vicinity for approximately a year without the need for additional control efforts. Initial conditions are found though a DC process targeting on the $\mathrm{x}$-axis velocity equal to zero (as in the $\mathrm{L}_{1}$ or $\mathrm{L}_{2}$ scenario). Figure 14 presents two different orbits, a small halo and a large Lissajous, similar to those used for the $\mathrm{L}_{1}$ and $\mathrm{L}_{2}$ analysis (see Table 1). As seen in the figure, the orbits are stable for the duration of the simulation - 250 days. When the orbit becomes unstable, either due to the dynamics or by a maneuver, the ensuing trajectory can be predicted by the computation of the Jacobi constant. Its natural motion after leaving the $\mathrm{L}_{3}$ location is to remain at about the Earth-Moon distance, but to evolve in a trajectory defined by zero-velocity curves.

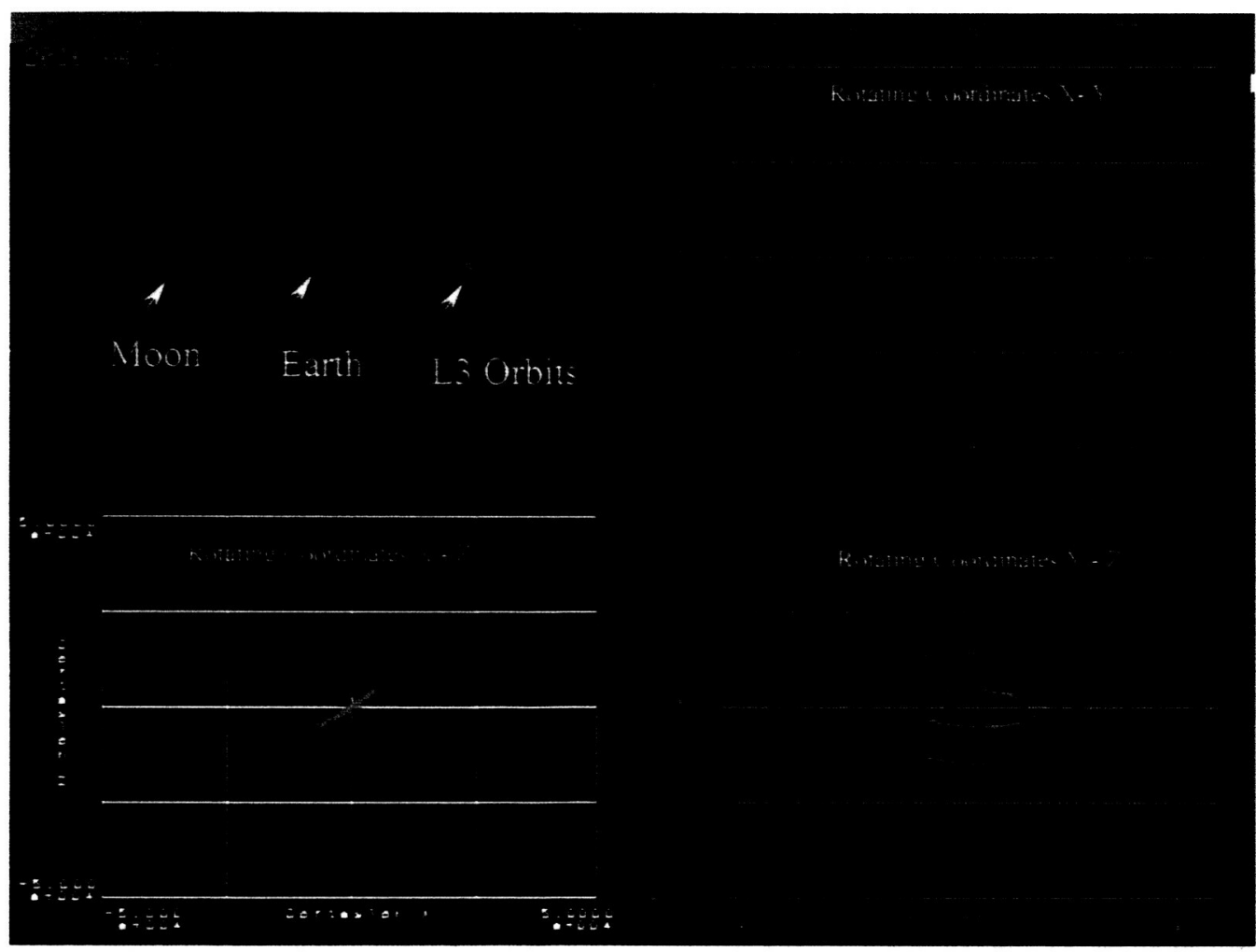

Figure 14. Colinear $\mathbf{L}_{3}$ Small halo and Large Lissajous Orbits

\section{C. $\mathrm{L}_{4} / \mathrm{L}_{5}$ Stationkeeping}

Stationkeeping about $\mathrm{L}_{4}$ and $\mathrm{L}_{5}$ is defined by the natural dynamics of a quasi-stable location. Orbits about these locations can remain stable for extended periods. One can use the Jacobi constant to define the region of interest or simply numerically integrate the equations of motion. These orbits do not require the same stationkeeping maintenance as those about $\mathrm{L}_{1}$ or $\mathrm{L}_{2}$, and are typically insensitive to mismodeling such as effects of errors in the SRP estimate. A peculiar property of the $\mathrm{L}_{4}$ and $\mathrm{L}_{5}$ locations is that motion about each location is determined by fundamental frequencies that define a long period and a short period. Several references have discussed this at length with explanations of the effects of the Earth-Moon mass ratio and solar gravity perturbation. ${ }^{2,3}$ Our goal is to find $\mathrm{L}_{4}$ and $\mathrm{L}_{5}$ orbits that meet the short term frequency (28 
day period) so that the motion of the orbit does not have a natural double frequency motion or escape the vicinity of the libration point. By judiciously selecting the initial conditions, an elliptical motion can be achieved by the elimination of the long period frequency. To accomplish this a differential correction scheme can be used to target the initial velocity vector to achieve the desired goal. We chose an initial condition that starts on the Earth- $\mathrm{L}_{4}$ (or equally $\mathrm{L}_{5}$ ) line with a velocity perpendicular to this line and in the direction of natural rotation to facilitate in attaining such an orbit. The target in this case is not the $\mathrm{x}$-axis velocity, but the $\mathrm{C} 3$ energy of the orbit with respect to the Earth. A C3 level of $-1.00 \mathrm{~km} / \mathrm{sec}^{2}$ minimizes the maximum elliptical distance and keeps the orbit motion about $\mathrm{L}_{4}$ or $\mathrm{L}_{5}$ for several years. Following the analysis by Michael, a maximum distance can be computed, but only for a CRTB system. ${ }^{2}$ Analysis using the effects of solar gravity and lunar eccentricity shows an expanding and contracting motion of the orbit. In reference 2 , the motion began at the $\mathrm{L}_{4}$ location itself. For our analysis, we assume that an orbit will be achieved after insertion from a transfer trajectory, so we used initial conditions similar to those of the $\mathrm{L}_{1}$ and $\mathrm{L}_{2}$ orbits, in both $\mathrm{L}_{4}$ centered $\mathrm{x}$ and $\mathrm{y}$ rotating coordinates (assuming EarthMoon rotating coordinate directions for the axes). The procedure for this initial condition selection can also be used for stationkeeping. Even with the targeted initial conditions, integrating with a full ephemeris model shows that the orbit will degrade and leave the $\mathrm{L}_{4}$ region, either from a lunar encounter or a change in the overall orbital energy due to third body accelerations or mismodeling.

A simple calculation of the $\Delta \mathrm{V}$ required to reset the $\mathrm{C} 3$ to the $-1.00 \mathrm{~km} / \mathrm{s}^{2}$ value was performed at an Earth apogee which yields an approximate $\mathrm{L}_{4} \mathrm{X}$-axis crossing. The angle with respect to the radial line to the Earth was chosen to be $\sim 90$ degrees. Initial velocity vector magnitudes and angles from the spacecraft-to-Earth line and the stationkeeping $\Delta \mathrm{Vs}$ to maintain $\mathrm{L}_{4}$ orbits are shown in Table 7 for several orbit amplitudes. Figures 15 and 16 show some sample orbits. The upper left shows the $\mathrm{L}_{4}$ orbit as it expands and contracts. The upper right is a polar plot of the

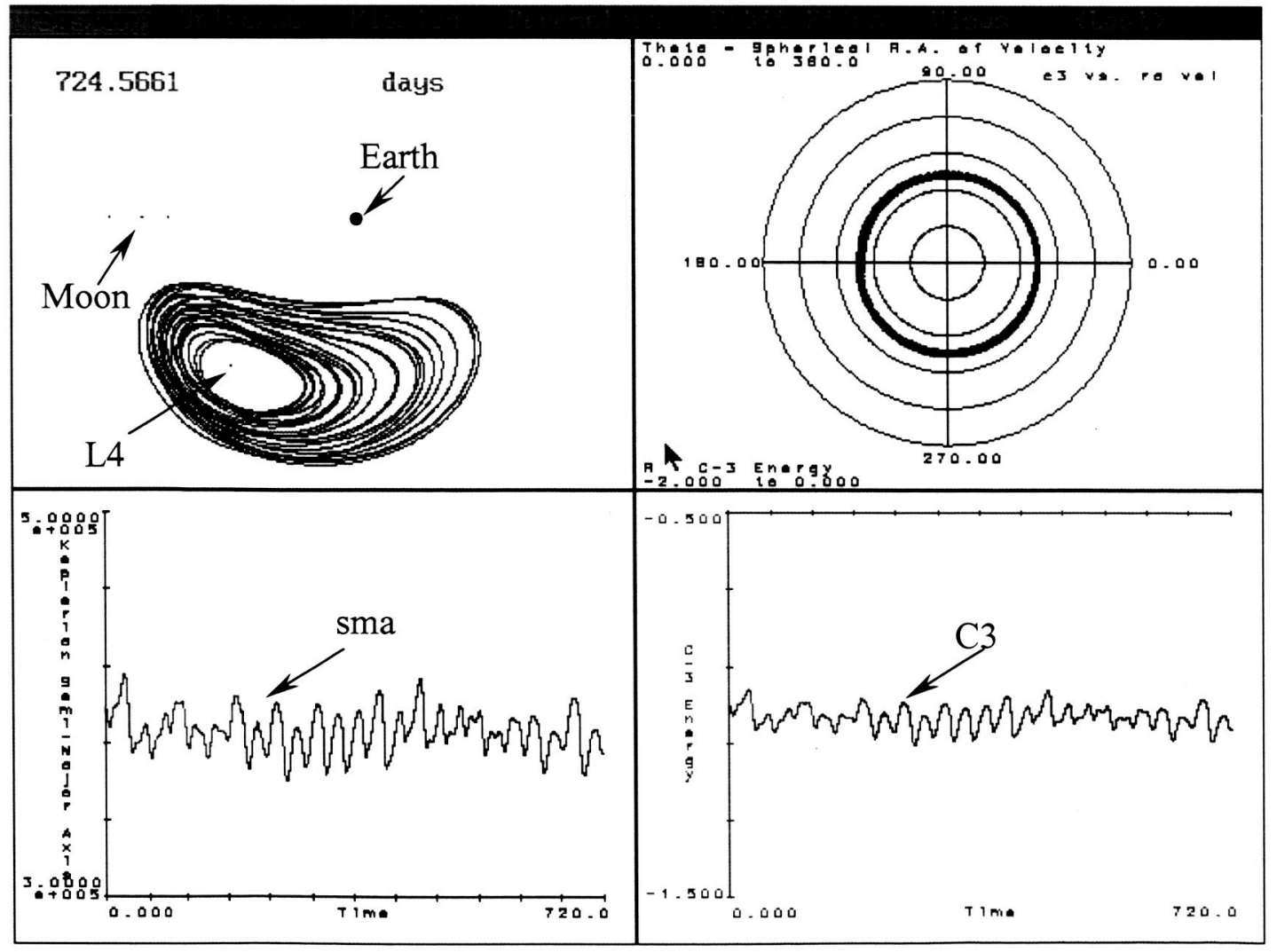

Figure 15. $\mathrm{L}_{4}$ Orbits, $\mathrm{C} 3$ and Semi-major Axis Information for Large Initial Amplitude 


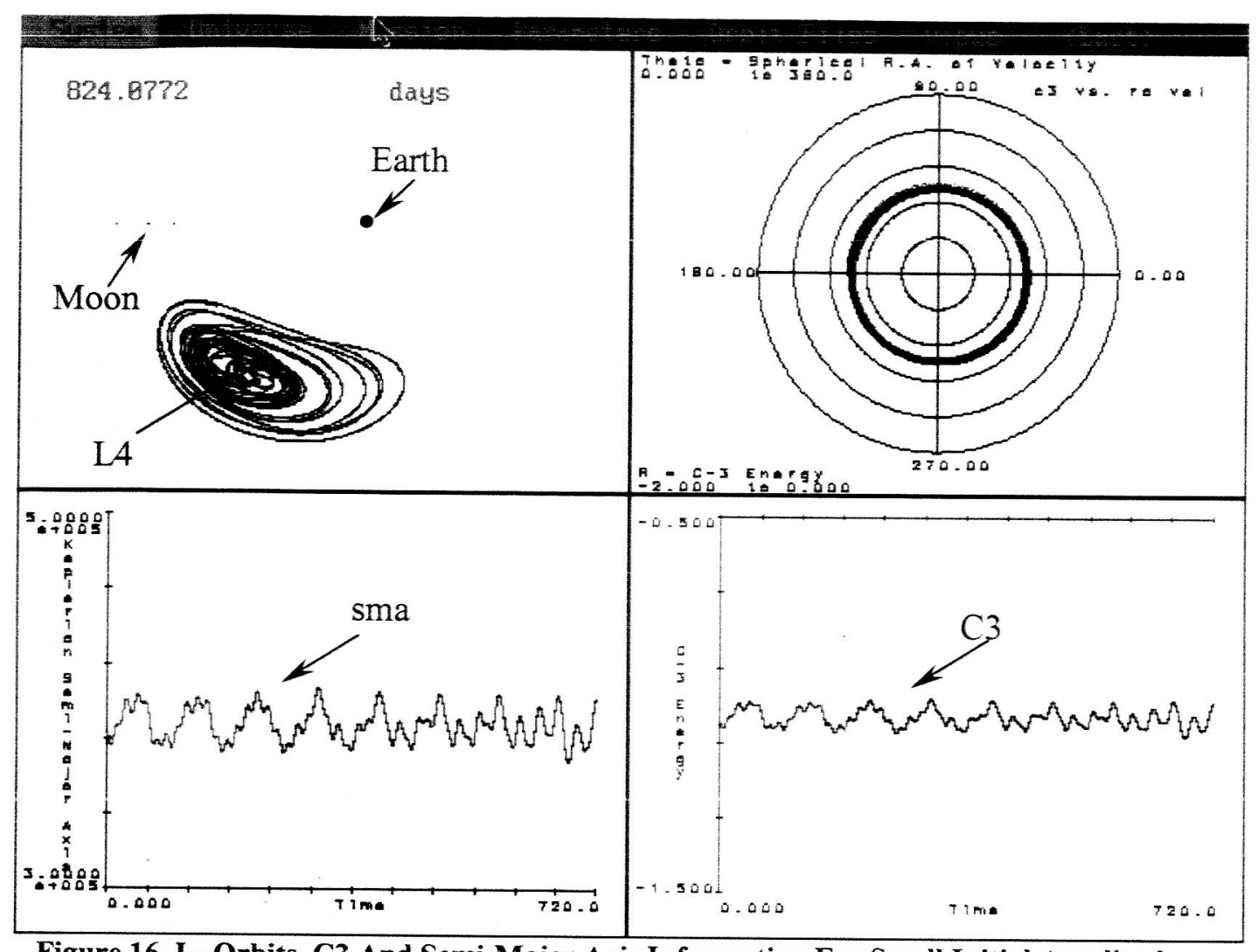

Figure 16. $\mathrm{L}_{4}$ Orbits, $\mathrm{C} 3$ And Semi-Major Axis Information For Small Initial Amplitude

C3 level versus the Right Ascension of the velocity. The lower left is the time history of the semi-major axis and the lower right is a time history of the C3.

Table 7. Yearly Stationkeeping $\Delta V$ for $L_{4}\left(L_{5}\right)$ Orbits Based on C3 Target

\begin{tabular}{|l|c|c|c|c|}
\hline $\begin{array}{c}\mathrm{X}-\mathrm{Y} \text { Starting } \\
\text { Position Relative } \\
\text { to } \mathrm{L}_{4}\end{array}$ & $\begin{array}{c}\text { Initial velocity } \\
\text { magnitude } \\
(\mathrm{km} / \mathrm{s}) \text { and angle } \\
\text { (deg) }\end{array}$ & $\begin{array}{c}\text { Stationkeeping } \\
\Delta \mathrm{V}(\mathrm{m} / \mathrm{s}) \text { and } \\
\text { angle }(\mathrm{deg})\end{array}$ & $\begin{array}{c}\text { Duration of } \\
\text { simulation (days) }\end{array}$ & $\begin{array}{c}\mathrm{C} 3\left(\mathrm{~km}^{2} / \mathrm{sec}^{2}\right) \\
\text { variation } \\
\text { (Earth centered } \\
\text { Mean Eq2000) }\end{array}$ \\
\hline${\text { At L } \mathrm{L}_{4}(0,0,0)}_{(5000,5000,0)}^{0.011 / 0}$ & $0.030 / 300$ & $29 / 270$ & 850 & $+/-0.03$ \\
\hline$(10000,10000,0)$ & $0.75 / 290$ & $57 / 270$ & 993 & $+/-0.03$ \\
\hline$(45000,45000,0)$ & $0.336 / 308$ & $31 / 270$ & 720 & $+/-0.03$ \\
\hline
\end{tabular}

\section{Transfer Results}

There obviously are an infinite number of possible transfers between libration orbits. Our numerical analysis looks at the most direct transfers that do not take us close to Earth (for radiation and shadow concerns) and that try to limit to a reasonable duration the transfer time. A target of less than a 28-day transfer was used, but as shown in the analysis, to minimize the $\Delta \mathrm{V}$ cost a natural motion is necessary which usually increases the transfer time duration. We used a $\mathrm{DC}$ process when possible and numerical integration (RK8/9) of the states along with full ephemeris and point mass gravity. 


\section{A. Co-linear to Co-linear}

A natural transfer between co-linear orbits is defined by the eigenstructure of the co-linear STM and the dynamics in question. Using the STM data generated in the above section for stationkeeping, an eigenvector map was generated and was plotted (Figure 2). As expected, for co-linear based orbits the unstable and stable modes are in the familiar directions and indicate that direct transfers between the co-linear locations are straightforward. That is, one uses the unstable mode of $\mathrm{L}_{1} / \mathrm{L}_{2}$ to perform a $\Delta \mathrm{V}$ (possibly of zero $\mathrm{m} / \mathrm{s}$ magnitude) to transfer to the stable mode of $\mathrm{L}_{2} / \mathrm{L}_{1}$ once the trajectory has moved to the other side of the secondary mass. This motion can be seen in Figures 17, 18, 19, 20, and 21 where a simple parametric scan was run that varied the time of a $10 \mathrm{~cm} / \mathrm{s}$ maneuver at a one day interval about the $\mathrm{L}_{1}$ or $\mathrm{L}_{2}$ orbits. Depending on the sign of the $\Delta \mathrm{V}$, the departure manifold either returned to the secondary mass, in this case the Moon, or went into orbit about Earth, or departed the Earth-Moon region.

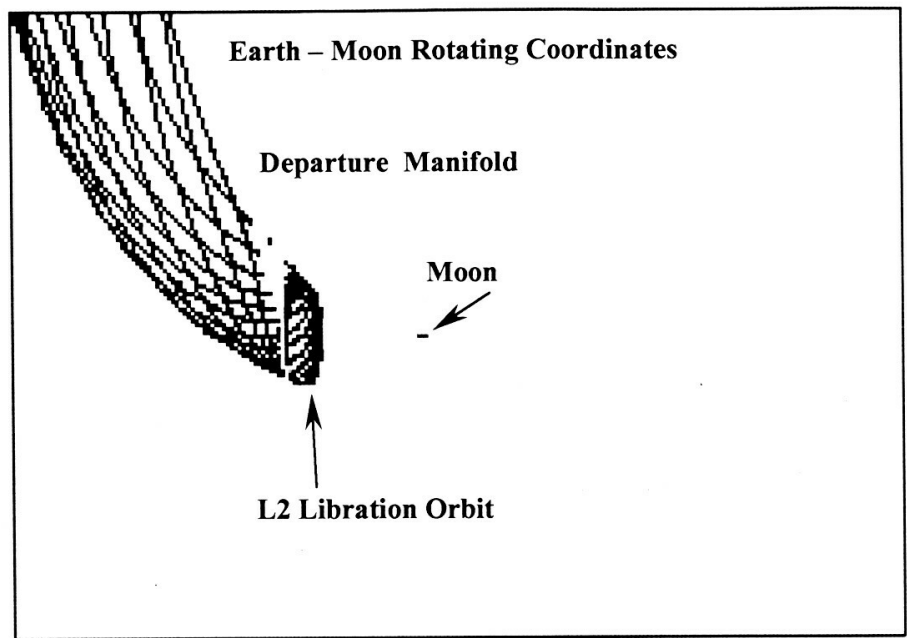

Figure 17. $\mathrm{L}_{2}$ Departure Manifold with an Increased Velocity

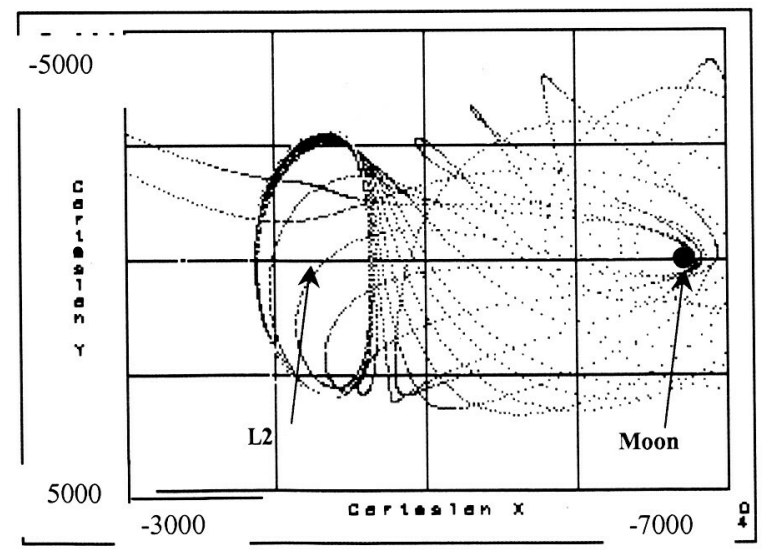

Figure 18. $\mathrm{L}_{2}$ Manifold with Velocity Decrease

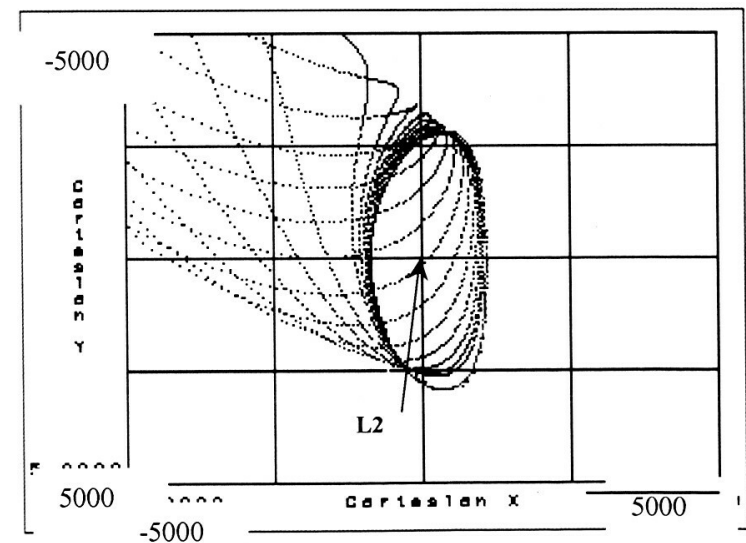

Figure 19. $L_{2}$ Manifold with Velocity Increase 


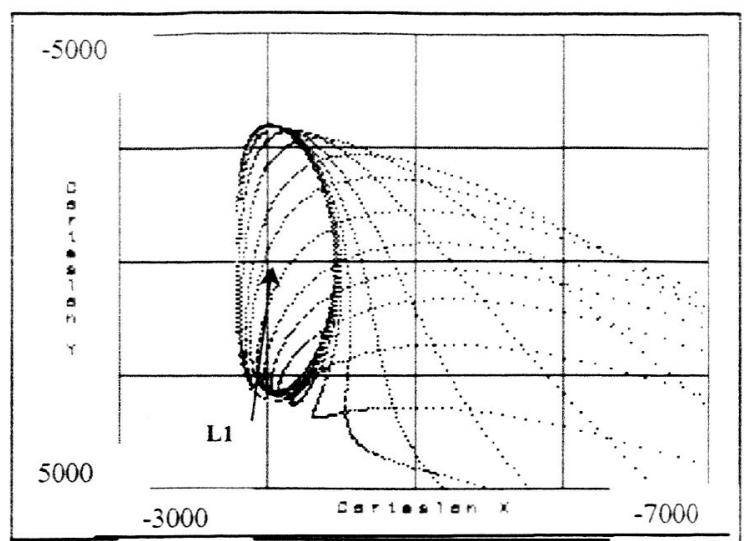

Figure 20. $L_{1}$ Manifold with Velocity Decrease

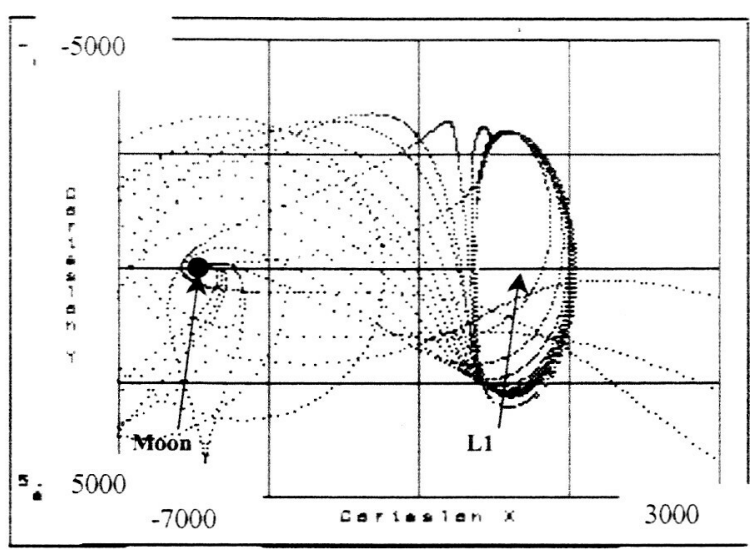

Figure 21. $\mathrm{L}_{1}$ Manifold with Velocity Increase

\section{B. $\mathbf{L}_{1}$ to $\mathbf{L}_{2}, \mathbf{L}_{3}$}

Applying a small $\Delta \mathrm{V}$ to change the velocity of the orbit in $\mathrm{L}_{1}$ centered coordinates we can perform the transfer from $\mathrm{L}_{1}$ to other co-linear locations. A small increase will guide the spacecraft into a trajectory that will pass by the secondary mass (Moon) and flip to the $\mathrm{L}_{2}$ side. The $\Delta \mathrm{V}$ to complete this transfer needs to be on the order of $0.1 \mathrm{~cm} / \mathrm{s}$ to ensure that the capture can be completed without placing the spacecraft on an outbound manifold into the Earth-Sun region or back towards Earth. Using a small $\Delta \mathrm{V}$ to decrease the velocity, the orbit will travel on the unstable manifold towards Earth and approach the $\mathrm{L}_{3}$ co-linear location. A $\Delta \mathrm{V}$ of $260 \mathrm{~m} / \mathrm{s}$ is required at perigee to adjust the apogee and an insertion $\Delta \mathrm{V}$ of approximately $220 \mathrm{~m} / \mathrm{s}$ will insert the spacecraft into a $L_{3}$ orbit. Figure 22 presents a sample trajectory for a transfer from $L_{1}$ to $L_{2}$. Figure 23 shows a 55-day transfer from $\mathrm{L}_{1}$ to $\mathrm{L}_{3}$.

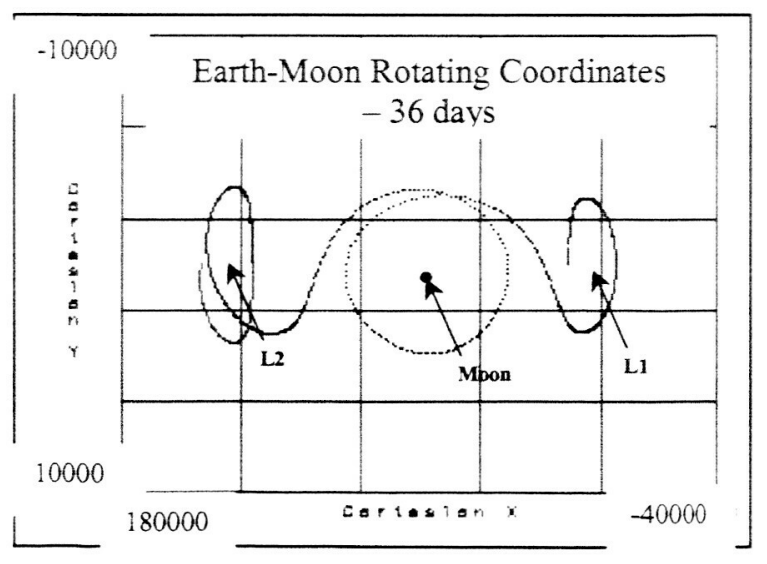

Figure 22. Transfer from $L_{1}$ to $L_{2}$

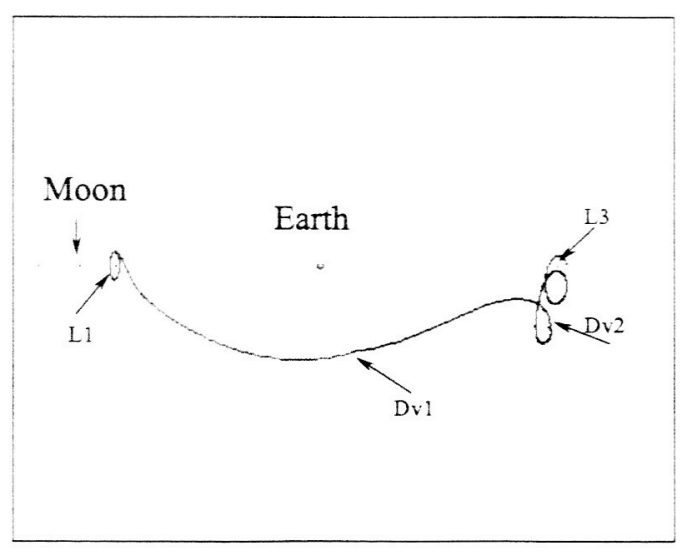

Figure 23. Transfer from $L_{1}$ to $L_{3}$ 


\section{C. $\mathrm{L}_{2}$ to $\mathrm{L}_{1}, \mathrm{~L}_{3}$}

Similar to the $\mathrm{L}_{1}$ scenario, a small $\Delta \mathrm{V}$ allows a transfer from $\mathrm{L}_{2}$ to the other co-linear locations. These transfers can be performed by applying a small $\Delta \mathrm{V}$ to decrease the velocity of the orbit in $\mathrm{L}_{2}$ centered coordinates. This decrease will guide the spacecraft into a trajectory that will pass by the secondary mass (Moon) and flip to the $\mathrm{L}_{1}$ side. The $\Delta \mathrm{V}$ to complete this transfer needs to be on the order of centimeters per second to ensure that the capture can be completed without placing the spacecraft on an inbound manifold towards the Earth. Once in orbit about $\mathrm{L}_{1}$, by using a small $\Delta \mathrm{V}$ to increase the velocity, the orbit will travel on the unstable manifold towards the Earth, swing past the Earth and approach the $\mathrm{L}_{3}$ co-linear location. At this point it is the same as the trajectory above. An insertion $\Delta \mathrm{V}$ of approximately $200 \mathrm{~m} / \mathrm{s}$ will insert the spacecraft into a $\mathrm{L}_{3}$ orbit.

\section{D. $\mathbf{L}_{3}$ to $\mathbf{L}_{1}, \mathbf{L}_{2}$}

An orbit about the Earth-Moon $\mathrm{L}_{3}$ location is quasi-stable and a small maneuver can place the spacecraft on a trajectory that will transfer it to $L_{1}$ or $L_{2}$. In Figures 24 and 25 , a small $1-\mathrm{m} / \mathrm{s}$ maneuver was used to initiate the transfer from the $\mathrm{L}_{3}$ orbit to the $\mathrm{L}_{2}$ location. Once there a small maneuver of approximately $55 \mathrm{~m} / \mathrm{s}$ can be used to capture into an orbit that will facilitate transfer to a smaller selected libration orbit. The transfer duration was 66 days, much higher than our desired goal of 28 days. A transfer to $\mathrm{L}_{1}$ from $\mathrm{L}_{2}$ required an additional $26 \mathrm{~m} / \mathrm{s}$.

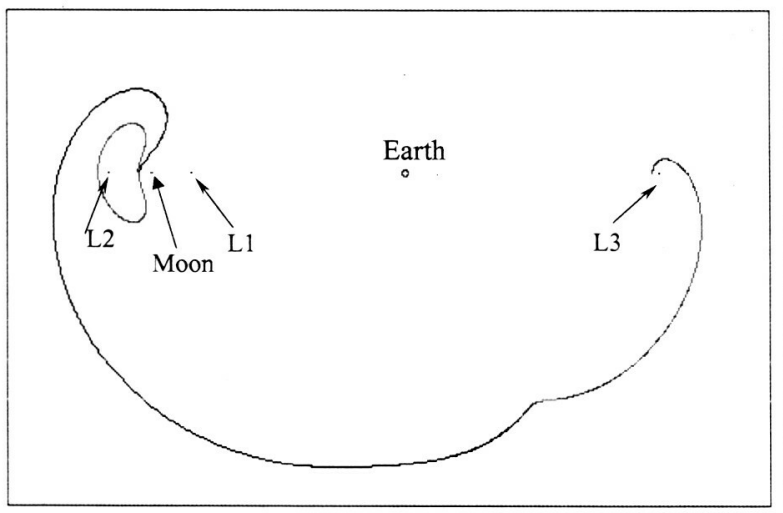

Figure 24. Transfer from $L_{3}$ to $L_{2}$

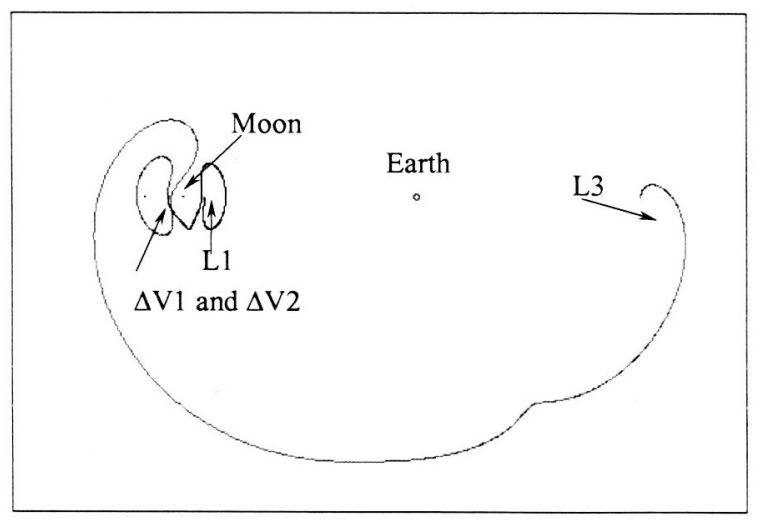

Figure 25. Transfer from $L_{3}$ to $L_{1}$

\section{E. Triangular to Triangular}

A natural transfer from triangular orbits is not defined by the eigen-information of the STM but by the dynamics in question. Using the information generated for stationkeeping in these regions, a Jacobi zero-velocity curve can be used to identify the trajectories of interest, keeping in mind the objective of minimizing the trip time. As expected, for triangular based orbits the zerovelocity curves are in the familiar directions and indicate that a direct transfer between the triangular locations is straightforward. That is, once a change in velocity has occurred, the spacecraft will 'drift' in the allowable regions until it approaches the other triangular location. This motion can be seen in Figure 26. Given enough time, the transfer from $L_{4}$ to $L_{5}$ or vice versa, can be achieved over an infinite selection of trajectories. Our goal is to minimize the trip time, so we limited our analysis to two-week trip times. A simple differential corrector scheme was used to target to the other triangular location once a trajectory profile that met the trip time requirement was found. Figure 26 shows a trajectory $L_{4}$ to $L_{5}$. The total $\Delta V$ was $228 \mathrm{~m} / \mathrm{s}(144$ $\mathrm{m} / \mathrm{s}$ for departure, $114 \mathrm{~m} / \mathrm{s}$ to capture) with a 45 -day transfer duration. 


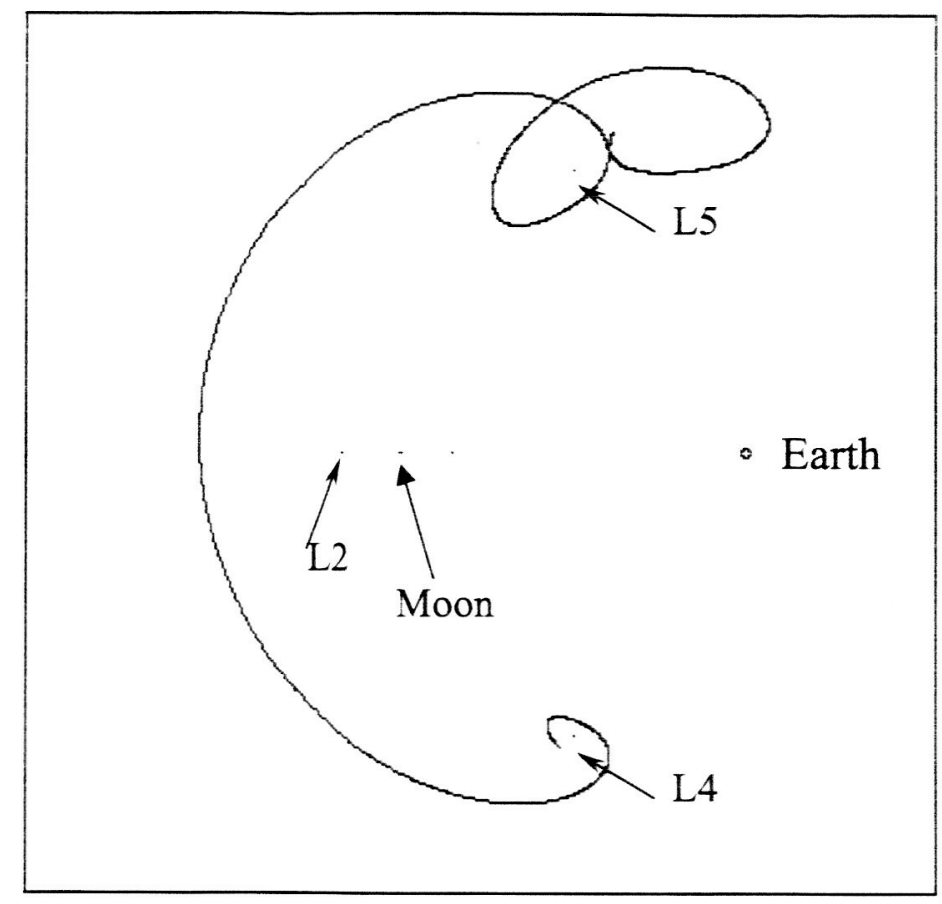

Figure 26. Transfer from $L_{4}$ to $L_{5}$

\section{F. Co-linear to Triangular}

Following the unstable modes of the $\mathrm{L}_{2}$ and $\mathrm{L}_{1}$ co-linear locations, it can be seen in Figure 17 that it is not possible to achieve a direct natural transfer from the co-linear to triangular locations by using the 'outbound' manifold. In fact it is not possible to achieve the transfer within a reasonable trip time (less than one month) without using the unstable mode that results in a trajectory that flows back towards the Earth. With judicious planning a single loop about the Earth, as viewed in the Earth-Moon rotating system, can be used to achieve the transfer. This results in other operational concerns such as shadows and transitions through radiation zones. Since this transfer requires greater than 28 days and resembles a transfer from the Earth to $\mathrm{L}_{4}$, it has not been included.

\section{G. Triangular to Co-linear}

The Jacobi constant can be used to determine the possible transfers from a triangular orbit to a colinear orbit and the allowable directions as defined by the zero-velocity curves. $\mathrm{A} \Delta \mathrm{V}$ is applied that increases the energy to a sufficient level to change the Jacobi constant that allows the transfer. Using the correct $\Delta \mathrm{V}$ and location for the maneuver, a trajectory can be computed that results in a close approach to the co-linear locations. A simple differential correction process can then be used to target to the co-linear orbit coordinates. The $\Delta \mathrm{V}$ for the $\mathrm{L}_{4}$ to $\mathrm{L}_{2}$ transfer required $288 \mathrm{~m} / \mathrm{s}$ total and a 30-day duration. The transfer from $L_{4}$ to $L_{1}$ required $460 \mathrm{~m} / \mathrm{s}$ and 138 days. Figures 27, 28, and 29 show transfers from $\mathrm{L}_{4}$ to $\mathrm{L}_{2}, \mathrm{~L}_{1}$, and $\mathrm{L}_{3}$. 


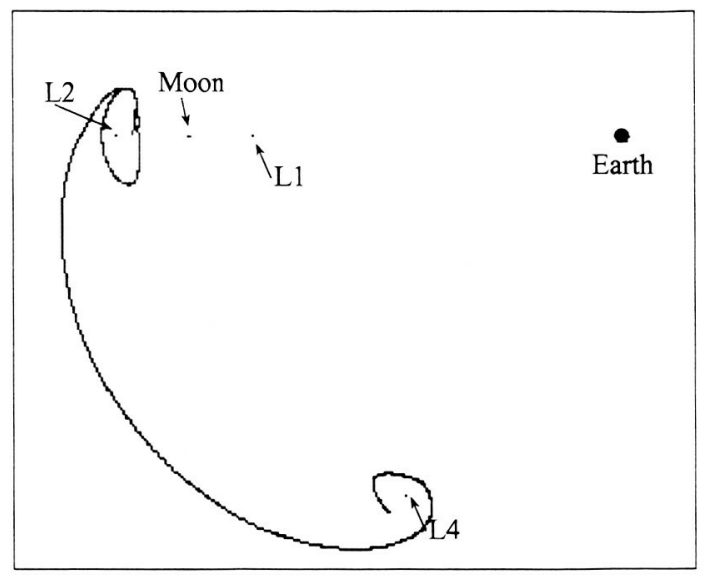

Figure 27. Transfer from $L_{4}$ to $L_{2}$

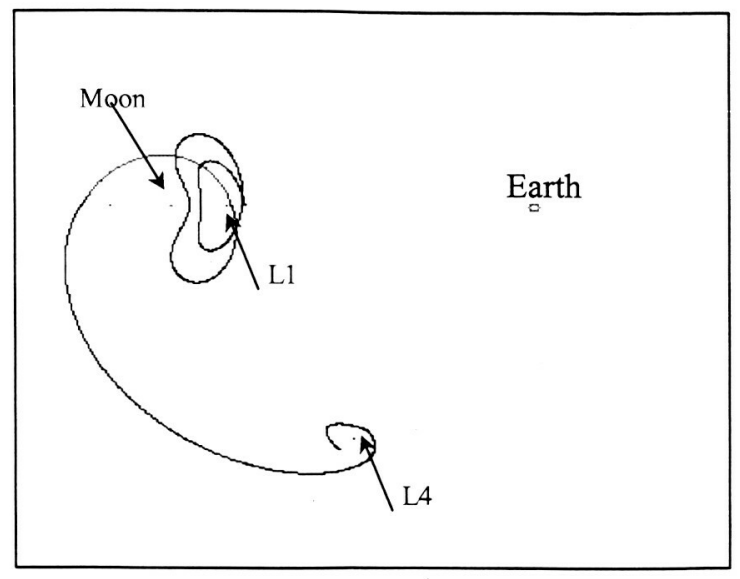

Figure 28. Transfer from $L_{4}$ to $L_{1}$

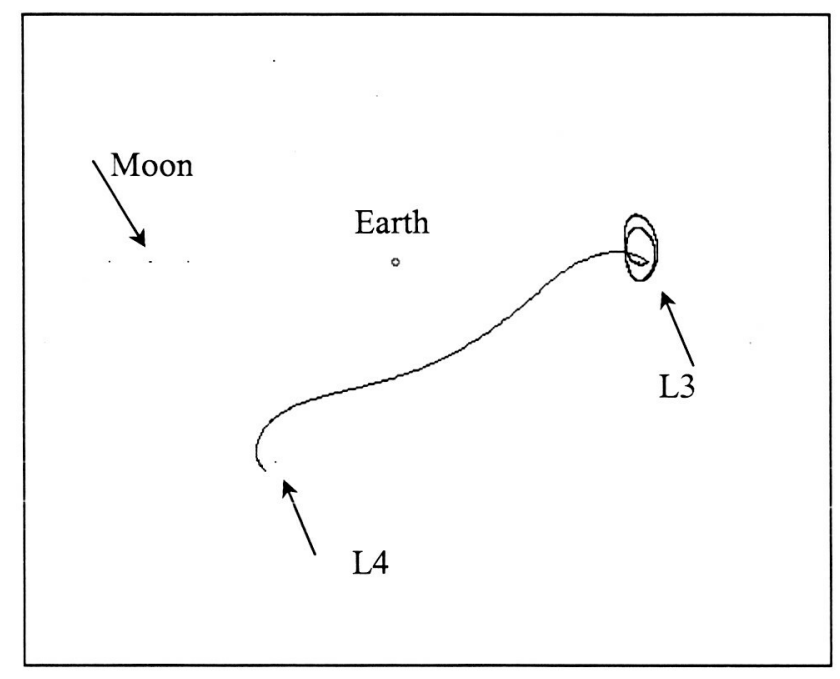

Figure 29. Transfer from $L_{4}$ to $L_{3}$

The $\Delta V$ s to effect the transfer from each libration location to another are listed in Table 8 along with the trip duration.

Table 8. Libration Orbit Cost for Transfers

\begin{tabular}{|l|c|c|c|c|c|}
\hline \multicolumn{1}{|c|}{ To } & $\mathbf{L}_{1}$ & $\mathbf{L}_{\mathbf{2}}$ & $\mathbf{L}_{3}$ & $\mathbf{L}_{\mathbf{4}}$ & $\mathbf{L}_{\mathbf{5}}$ \\
\hline $\mathbf{L}_{\mathbf{1}}$ & $\mathrm{x}$ & $<1 \mathrm{~m} / \mathrm{s}-14 \mathrm{~d}$ & $480 \mathrm{~m} / \mathrm{s}-55 \mathrm{~d}$ & $\mathrm{a}$ & $\mathrm{b}$ \\
\hline $\mathbf{L}_{2}$ & $<1 \mathrm{~m} / \mathrm{s}-14 \mathrm{~d}$ & $\mathrm{x}$ & $480 \mathrm{~m} / \mathrm{s}>55 \mathrm{~d}$ & $\mathrm{c}$ & $\mathrm{d}$ \\
\hline $\mathbf{L}_{3}$ & $102 \mathrm{~m} / \mathrm{s}-90 \mathrm{~d}$ & $55 \mathrm{~m} / \mathrm{s}-66 \mathrm{~d}$ & $\mathrm{x}$ & $73 \mathrm{~m} / \mathrm{s}-58 \mathrm{~d}$ & $\mathrm{e}$ \\
\hline $\mathbf{L}_{4}$ & $460 \mathrm{~m} / \mathrm{s}-138 \mathrm{~d}$ & $288 \mathrm{~m} / \mathrm{s}-30 \mathrm{~d}$ & $752 \mathrm{~m} / \mathrm{s}-28 \mathrm{~d}$ & $\mathrm{x}$ & $228 \mathrm{~m} / \mathrm{s}-45 \mathrm{~d}$ \\
\hline $\mathbf{L}_{5}$ & $\mathrm{e}$ & $\mathrm{e}$ & $\mathrm{e}$ & $228 \mathrm{~m} / \mathrm{s}-45 \mathrm{~d}$ & $\mathrm{x}$ \\
\hline
\end{tabular}

$\mathrm{a}=$ time required exceeds 28 days

$\mathrm{b}=$ time required exceeds 28 days

$\mathrm{c}=$ transfer not possible

$\mathrm{d}=$ transfer not possible

$\mathrm{e}=$ similar to $\mathrm{L}_{4}$ transfers 


\section{Summary}

Stationkeeping cost is dependent upon the amplitude of the libration orbit, the perturbations included, and the errors associated with operations, which include navigation errors. In our analysis, the $L_{1}$ and $L_{2}$ stationkeeping cost without errors has a floor of about $10 \mathrm{~m} / \mathrm{s}$ per year. This minimum is due to the method of selecting our targeting goals. We used a goal of zero velocity in the $+x$-axis direction for consistency in all simulations with maneuvers performed every half-orbit period. The correct velocity targets will be slightly different, possibly closer to $+/-10 \mathrm{~m} / \mathrm{s}$ in the $\mathrm{x}$-axis direction. Also, a multiple revolution target reduces the no-error $\Delta \mathrm{V}$ cost to just a few $\mathrm{m} / \mathrm{s}$ per year, but the addition of the navigation, SRP, and maneuver errors drive the $\triangle V$ budget to much higher levels. The $D C$ targeting method provides a lower cost than the $\mathrm{dLQR}$ for the base Lissajous orbits as it targets a condition that balances the orbit and allows it to complete another revolution without a reference orbit. This method may be similar to the removal of the unstable mode at specific intervals, but is dependent upon the target selection criteria. The stationkeeping of a halo orbit is more costly, but will depend upon the method to maintain the $z$-component. We analyzed the cost of controlling the $z$-axis with a maneuver in the RIP $z$-axis direction at 1.5 days after the balancing maneuver performed at the $x-z$ plane crossing. Also of note is that the $\mathrm{DC}$ targeter required an additional $\Delta \mathrm{V}$ to provide a reasonable initial two-to-three orbits before settling in on smaller corrections, as seen in the figures. Stationkeeping of the $\mathrm{L}_{3}, \mathrm{~L}_{4}, \mathrm{~L}_{5}$ locations was found to be of minimal cost, but still required some assistance. A goal of maintaining a constrained $\mathrm{L}_{4}$ orbit size seems unmanageable from a $\Delta V$ cost perspective, as the control must cancel much of the natural orbital dynamics. The yearly $\Delta V$ cost for $\mathrm{L}_{4}$ and $\mathrm{L}_{5}$ stationkeeping is on the order of tens of meters per second, and can easily be accomplished by targeting a $\mathrm{C} 3$ value of $-1.0 \mathrm{~km}^{2} / \mathrm{s}^{2}$. The $\Delta \mathrm{V}$ costs for transfer between libration orbit locations is dependent on the trip time and the use of natural motions which follow the zerovelocity contours. The transfer $\Delta \mathrm{V}$ costs range from tens of meters per second to several hundred meters per second.

\section{Conclusions}

Stationkeeping costs at the Earth-Moon system libration points cannot be estimated using simple Circular Restricted Three Body motion but must address the total N-body dynamics. Use of a full ephemeris and operational errors should be included in deriving any cost estimate. The dynamics of the region should be more fully understood, such as the differences between the $\mathrm{L}_{1}$ and $\mathrm{L}_{2}$ regions, in order to select the correct target goals when using a differential corrector for these stationkeeping estimates. The stationkeeping estimates of the quasi-stable $L_{4}, L_{5}$ and $L_{3}$ locations were shown to be low and can be simplified by using common two-body and RLP plane crossing targets. Transfers between the Earth-Moon libration locations are very dependent upon the overall dynamics. The use of natural motions may reduce $\Delta \mathrm{V}$ cost, but may significantly increase trip durations.

\section{References}

1. V. Szebehely, Theory of Orbits, The Restricted Problem of Three Bodies, Academy Press, 1967

2. L. Steg and J. deVries, Earth-Moon Libration Points: Theory, Existence, and Applications, "Space Sci, Rev 5, pp 210-233, 1966

3. W. Michael, "Considerations of the Motion of a Small Body in the Vicinity of the Stable Libration Points of the Earth-Moon System", NASA TR R-160, Langley Research Center, Hampton, Va., 1963 
4. R. Farquhar, "The Utilization of Halo Orbits in Advanced Lunar Operations", NASA TN D6365, GSFC, Greenbelt, MD., 1971

5. D. Hoffman, "Stationkeeping at the Colinear Equilibrium Points of the Earth-Moon System", NASA JSC-26189, Sept. 1993

6. C. Renault and D. Scheeres, "Statistical Analysis of Control Maneuvers in Unstable Orbital Environments," Journal of Guidance, Control, and Dynamics, Vol 26, No. 5, Sept-Oct 2003

7. K.C. Howell and T. Keeter, "Stationkeeping Strategies for Libration Point Orbits - Target Point and Floquet Mode Approaches," Advances in Astronautical Sciences, Vol 89, Pt 2, pp.1377-1396, 1995

8. C. Jacobi, Vorlesungen uber Dynamik, Reimer Publisher, Berlin, 1866

9. B. Marchand and K. Howell, "Formation Flight Near L1 and L2 in the Sun-Earth-Moon Ephemeris System Including Solar Radiation Pressure, AAS/AIAA Astrodynamics Specialist Conference, Big Sky Montana, Aug. 2003

10. D. Folta, et al, "Servicing and Deployment of National Resources in Sun-Earth Libration Point Orbits", $53^{\text {rd }}$ International Astronautical Congress, World Space Congress, Houston, TX, Oct 10-19, 2002

11. N. Hamilton and D. Folta, "Formation Flying Satellite Control Around the L2 Sun-Earth Libration Point", AAS/AIAA Astrodynamics Specialist Conference, Monterey CA, August 2002

12. P. Dorato, et al, Linear-Quadratic Control, An Introduction, Prentice Hall, 1995

13 D. Folta, Swingby Mathematical Principles, 553-FDD-92/058R1UD0 Rev 1, Sept. 1995,

14 D. Folta "Libration Orbit Mission Design: Application of Numerical and Dynamical Methods", Libration Point Orbits and Applications, Parador d'Aiguablava, Girona, Spain, June $10-14,2002$

15 F. Abilleira, "Automating Astrogator: An Astrogator / Connect Reference", Internal memo, NASA/ GSFC, July 2003 